The online version of this article is published within an Open Access environment subject to the conditions of the Creative Commons Attribution-NonCommercial-ShareAlike licence $<$ http://creativecommons.org/licenses/by-nc-sa/3.0/ $>$. The written permission of Cambridge University Press must be obtained for commercial re-use.

doi: $10.1017 / \mathrm{jfm} .2013 .147$

\title{
Drop impact entrapment of bubble rings
}

\author{
M.-J. Thoraval ${ }^{1}$, K. Takehara ${ }^{2}$, T. G. Etoh ${ }^{2}$ and S. T. Thoroddsen ${ }^{1, \dagger}$ \\ ${ }^{1}$ Division of Physical Sciences and Engineering and Clean Combustion Research Center, King Abdullah \\ University of Science and Technology (KAUST), Thuwal 23955-6900, Saudi Arabia \\ ${ }^{2}$ Department of Civil and Environmental Engineering, Kinki University, Higashi-Osaka 577-8502, Japan
}

(Received 7 August 2012; revised 26 January 2013; accepted 7 March 2013; first published online 29 April 2013)

We use ultra-high-speed video imaging to look at the initial contact of a drop impacting on a liquid layer. We observe experimentally the vortex street and the bubble-ring entrapments predicted numerically, for high impact velocities, by Thoraval et al. (Phys. Rev. Lett., vol. 108, 2012, article 264506). These dynamics mainly occur within $50 \mu$ s after the first contact, requiring imaging at 1 million f.p.s. For a water drop impacting on a thin layer of water, the entrapment of isolated bubbles starts through azimuthal instability, which forms at low impact velocities, in the neck connecting the drop and pool. For Reynolds number Re above $\sim 12000$, up to 10 partial bubble rings have been observed at the base of the ejecta, starting when the contact is $\sim 20 \%$ of the drop size. More regular bubble rings are observed for a pool of ethanol or methanol. The video imaging shows rotation around some of these air cylinders, which can temporarily delay their breakup into micro-bubbles. The different refractive index in the pool liquid reveals the destabilization of the vortices and the formation of streamwise vortices and intricate vortex tangles. Fine-scale axisymmetry is thereby destroyed. We show also that the shape of the drop has a strong influence on these dynamics.

Key words: breakup/coalescence, drops and bubbles

\section{Introduction}

The impact of a drop on a pool surface has been studied for over a century, but revolutionary improvements in high-speed video technology (Etoh et al. 2003) have recently opened up this canonical geometry to renewed scrutiny. This applies especially to the earliest contact between the drop and the pool, where intricate details have emerged and play a crucial role during air entrapment and splashing (Yarin 2006; Thoroddsen, Etoh \& Takehara 2008).

The impact of a drop always entraps a bubble under the centre of the drop, as a disk of air is produced by the lubrication pressure and rapidly contracts into a bubble at the centre (Thoroddsen, Etoh \& Takehara 2003; Liow \& Cole 2007; Korobkin, Ellis \& Smith 2008; Hicks \& Purvis 2010; Mani, Mandre \& Brenner 2010; Driscoll \& Nagel 2011; Kolinski et al. 2012; van der Veen et al. 2012). Following this central 
air disk entrapment on a liquid pool, the outer contact forms a neck, which emits an ejecta sheet for sufficiently large Reynolds numbers (Weiss \& Yarin 1999; Davidson 2002; Thoroddsen 2002; Josserand \& Zaleski 2003; Howison et al. 2005). These ejecta are the source of the finest spray droplets (Thoroddsen et al. 2011; Zhang et al. 2012), which is of relevance to numerous processes, such as combustion and aerosol formation.

However, at even larger impact energy, these smooth ejecta give way to random splashing of small droplets, see Thoroddsen (2002). Numerical simulations by Thoraval et al. (2012) have shown that the base of the ejecta can become unstable, bending up and down as the free surface sheds alternate-sign vortex rings into the liquid, and often entrapping bubble rings. These bubble rings alternate between the top and bottom of the ejecta. This air entrapment has important applications for inkjet printing and gas transfer at the liquid-air interface (Wanninkhof et al. 2009; Czerski et al. 2011), as it can generate hundreds of micro-bubbles. This regime is the focus of the current investigation.

Very recent experiments by Castrejón-Pita, Castrejón-Pita \& Hutchings (2012) have used side-view and laser-induced fluorescence to verify the presence of the von Kármán street for conditions similar to those in Thoraval et al. (2012). Herein we show the first experimental observations of the formation and breakup of the bubble tori.

The main axisymmetric features of the vortex street and bubble-ring entrapments are observed experimentally. However, three-dimensional effects rapidly break the axisymmetry. Herein, we show that even at rather modest impact velocities, azimuthal instabilities can appear in the neck between the drop and the pool. Imaging using two different liquids also reveals the shedding of streamwise vortices and their intricate dynamics, similar to three-dimensional instabilities of the cylinder wake (Williamson 1996), or the shear layer (Lasheras \& Choi 1988). These intricate structures have perhaps escaped earlier experimental notice as they develop in a sub-millimetre region and evolve in less than $50 \mu \mathrm{s}$.

\section{Experimental setup and numerics}

\subsection{High-speed video imaging}

In this work we image drop impacts on shallow pools through a bottom glass plate (figure 1). Limited imaging (only in figure 15a,b) was done from the side above the pool surface. We use identical water drops in the entire study, while changing the composition of the pool liquid. The pool liquids tested are water, ethanol and methanol which are all highly miscible with the water drop. The liquid properties are given in table 1. The difference in refractive index between the water drop and the ethanol or methanol pools allows us to image the flow structures as they distort the interface between the two liquids.

The use of shallow pools, or thin films, is dictated by the need to change the pool liquid following every impact as well as by the optical setup, where the limited focal distance of the long-distance microscope rules out bottom views through deep pools. The liquid was contained by a $10 \mathrm{~cm}$ diameter ring glued onto the glass plate. The pool depth $\delta$ was varied from $\sim 25 \mu \mathrm{m}$ to $1 \mathrm{~mm}$. It was estimated by controlling the volume of liquid with a syringe and assuming a uniform spreading inside the container. To minimize the effect of evaporation, the impact experiment was done within $10 \mathrm{~s}$ after spreading the liquid. 
(a)

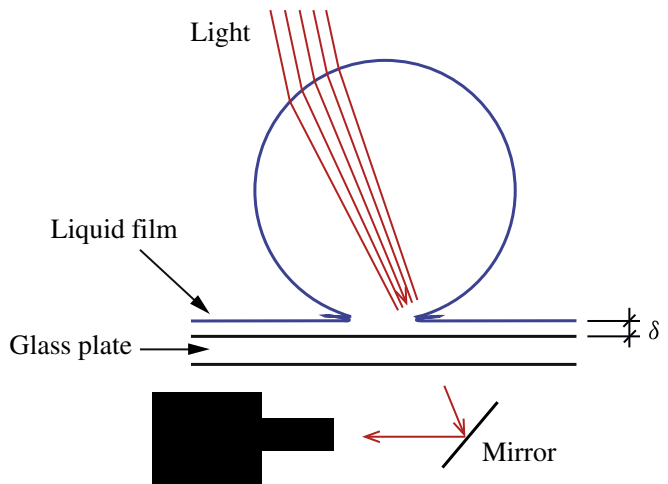

(b)

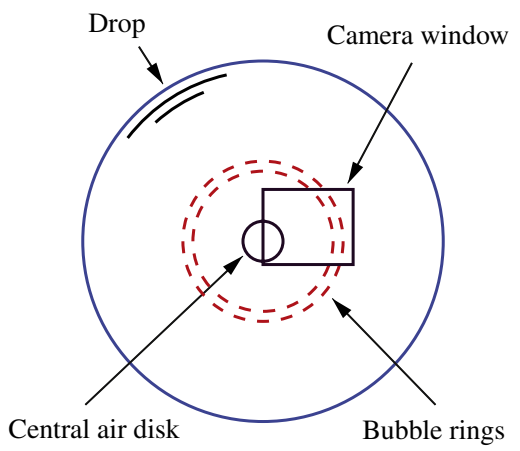

High-speed camera

FIGURE 1. (a) Imaging setup. We use backlight imaging of the drop impact from below, through a glass plate. The drop acts as a lens focusing the illumination to a limited area on the observation window. Several lights were used in some cases to obtain a larger illuminated area. $(b)$ Camera viewing area shown in bottom view, corresponding to the area in figure $10(c)$.

$\begin{array}{lccccc}\text { Liquid } & \rho\left(\mathrm{g} \mathrm{cm}^{-3}\right) & \mu(\mathrm{cP}) & v(\mathrm{cSt}) & \sigma\left(\text { dyne } \mathrm{cm}^{-1}\right) & n \\ \text { Distilled water } & 0.996 & 1.004 & 1.008 & 72.1 & 1.333 \\ \text { Ethanol } & 0.789 & 1.19 & 1.51 & 23.2 & 1.363 \\ \text { Methanol } & 0.793 & 0.593 & 0.748 & 22.5 & 1.339\end{array}$

TABLE 1. Properties of the different liquids used in the pool. Here $\rho$ is the liquid density; $\mu$ is the dynamic viscosity; $v$ is the kinematic viscosity, $n$ the refractive index at $\lambda=532 \mathrm{~nm}$ and $\sigma$ the surface tension. The drop is always water.

Here we use a long-distance microscope for magnifications up to $\sim 15$ for maximum pixel resolution of $\sim 4.1 \mu \mathrm{px}^{-1}$, when using a Shimadzu Hypervision CCD video camera (Etoh et al. 2003), at frame rates up to 1 million f.p.s. Some of the imaging was also done at a lower frame rate with a Photron SA5 CMOS camera, with a magnification up to 10 and maximum pixel resolution $\sim 2 \mu \mathrm{m} \mathrm{px}^{-1}$. Using thin bottom layers restricts the vertical motion of the interface between the drop and the pool liquid during the impact, thereby making well-focused imaging easier with the limited focal depth. For further optical/triggering details see Thoroddsen, Takehara \& Etoh (2012).

The drop is pinched from a $3 \mathrm{~mm}$ nozzle, to produce an effective drop diameter of $D=\left(D_{v} D_{h}^{2}\right)^{1 / 3}=4.67 \mathrm{~mm}$, where $D_{v}$ and $D_{h}$ are the instantaneous vertical and horizontal diameters. We characterize the impact conditions by the Reynolds number $R e$, the Weber number $W e$ and the splashing parameter $K$, defined as:

$$
R e=\frac{\rho D V}{\mu}, \quad W e=\frac{\rho D V^{2}}{\sigma}, \quad K=W e \sqrt{R e},
$$

where $\rho, \mu$ and $\sigma$ are respectively the density, dynamic viscosity and surface tension of the drop liquid, and $V$ the drop impact velocity. 


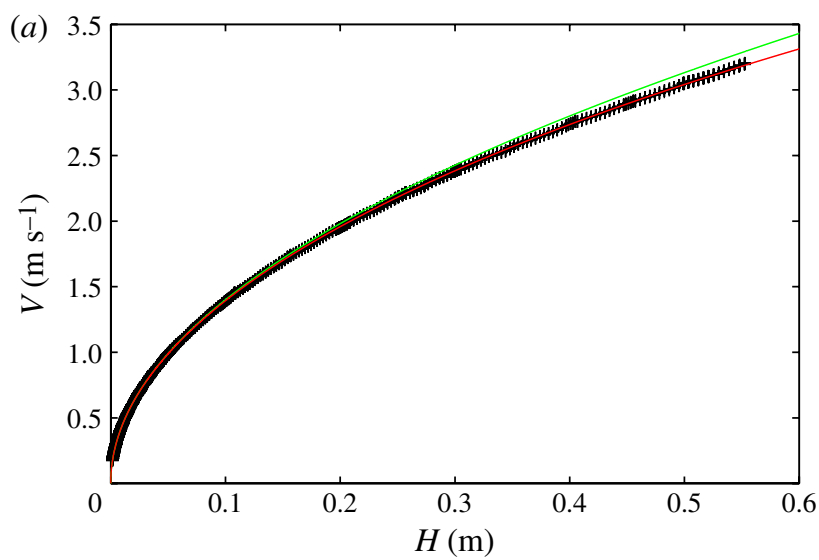

(b)

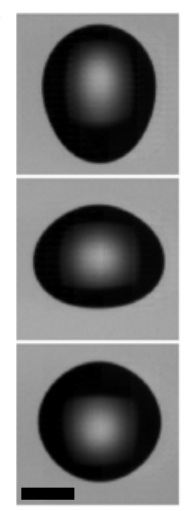

FIGURE 2. (a) Drop velocity $V$ versus falling height $H$. The green line corresponds to $\sqrt{2 g H}$, while the red line is our fitting equation. (b) Typical drop shapes in air: prolate $(\alpha>1$, $H=31.2 \mathrm{~cm}, \operatorname{Re}=11300)$, oblate $(\alpha<1, H=41.8 \mathrm{~cm}, \operatorname{Re}=12900)$ and close to spherical ( $\alpha \simeq 1, H=44.5 \mathrm{~cm}, R e=13300$ ). The scale bar is $2 \mathrm{~mm}$ long.

The drop velocity $V$ was characterized in a separate set of experiments. It was then modelled by the velocity of a sphere experiencing constant drag (Pumphrey, Crum \& Bjørnø1989; Saylor \& Grizzard 2004):

$$
V=V_{T} \sqrt{1-\exp \left(-\frac{2 g\left(h-D-h_{0}\right)}{V_{T}^{2}}\right)},
$$

where gravity is $g=9.81 \mathrm{~m} \mathrm{~s}^{-2}$, with the fitting parameters $V_{T}=9.11 \mathrm{~m} \mathrm{~s}^{-1}$ and $h_{0}=2.1 \mathrm{~mm}$. $V_{T}$ corresponds to the terminal velocity of the drop, and $h_{0}$ to the effective pinch-off length of the drop when it separates from the nozzle. Here $h$ is the measured distance from the nozzle tip to the undisturbed pool surface, whereas the adjusted height is defined as $H=h-D-h_{0}$. Figure 2(a) shows that the measured values of $V$ deviate by less than $0.8 \%$ from the formula, for our impact heights $2.5 \mathrm{~cm}<H<55 \mathrm{~cm}$. This estimate of $V_{T}$ is slightly higher than the experimental observations of Gunn \& Kinzer (1949), which could be due to the drop oscillations before reaching a final oblate shape. We can calculate the falling time of the drop from the falling height as: $t=\left(V_{T} / g\right) \arg \cosh \left[\exp \left(g H / V_{T}^{2}\right)\right]$.

As $D$ is larger than the capillary length for water, $l_{c}=\sqrt{\sigma /(\rho g)}=2.7 \mathrm{~mm}$, the water drop shows large oscillations that can affect the details of the impact dynamics (see figure $2 b$ ). The axisymmetric vertical oscillations of the drop can be estimated by the dominant mode (Rayleigh 1879; Lamb 1975, §275), giving a radius:

$$
R(t, \theta)=R_{0}\left[1+a \cos (\omega t+\phi) P_{2}(\cos \theta)\right],
$$

where $P_{2}(x)=\left(3 x^{2}-1\right) / 2$ is the Legendre polynomial of degree two, and $\theta$ is the polar angle in the spherical coordinate system. The aspect ratio between the vertical and horizontal diameters of the drop can thus be written:

$$
\alpha=\frac{D_{v}}{D_{h}}=\frac{1+a \cos (\omega t+\phi)}{1-\frac{a}{2} \cos (\omega t+\phi)} .
$$




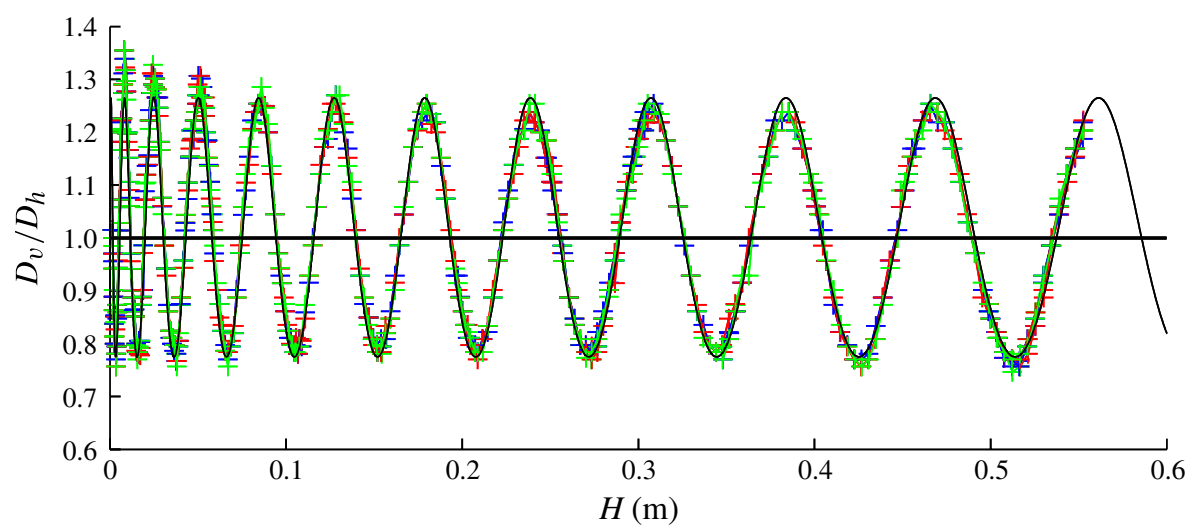

FIGURE 3. Drop aspect ratio $\alpha=D_{v} / D_{h}$ versus $H$. The black oscillating line corresponds to the fitted equation (2.4). Different colours denote different realizations.

We determine $a, \omega$ and $\phi$ as fitting parameters: $a=0.162, f=\omega /(2 \pi)=33.2 \mathrm{~Hz}$ and $\phi=-132^{\circ}$. The oscillation frequency is only $2 \%$ lower than the inviscid theoretical value $f_{D}=(4 / \pi) \sqrt{\sigma /\left(\rho D^{3}\right)}=33.9 \mathrm{~Hz}$. The typical time of bubble-ring entrapment, $50 \mu \mathrm{s}$, is only $0.17 \%$ of the oscillation period. Therefore the drop shape can be considered frozen during the entrapment. This fitting is then used to obtain the aspect ratio from the falling height in the experiments. We have neglected viscous damping of the dominant mode in this estimate of drop oscillations. The characteristic time of this damping can be estimated as $\tau=D^{2} /(20 v)=1.08 \mathrm{~s}$ (Lamb 1975, §355). In the overall falling time studied here $(\simeq 0.35 \mathrm{~s})$, viscous effects can be estimated to reduce the amplitude of the dominant mode by $27 \%$. It is therefore too short to damp the oscillations significantly, as is observed in figure 3 .

\subsection{Numerical method}

We use the open-source software Gerris (http://gfs.sf.net; Popinet 2003, 2009; Agbaglah et al. 2011), using the Volume-Of-Fluid method, to perform axisymmetric simulations of the drop impacts. The liquid from the drop and the pool are identical but identified with different markers (drop: red, pool: blue, air: light green). The adaptive mesh is refined dynamically based on the distance to the interface, vorticity magnitude and geometric conditions. The interface is refined uniformly at the maximum level in the simulations. The bubbles and droplets with area less than 10 cells are removed during the computation, as their dynamics cannot be captured accurately. It represents an effective cut-off diameter of $D_{\text {cut }}=3.57$ cells.

The simulations are started with the drop $0.1 R$ above the pool, where $R=D / 2$ is the drop radius. Non-dimensional time is defined as $t^{*}=t / \tau$, where $\tau=D / V$. The origin of time is taken when the undisturbed sphere would first contact the pool. The drop is kept at a constant effective diameter $D=4.6 \mathrm{~mm}$. Air has a viscosity of $\mu_{a}=1.81 \times 10^{-2} \mathrm{cP}$ and density $\rho_{a}=1.21 \mathrm{~kg} \mathrm{~m}^{-3}$. The liquid is water for both the drop and the pool, with viscosity $\mu=1 \mathrm{cP}$, density $\rho=1000 \mathrm{~kg} \mathrm{~m}^{-3}$ and surface tension $\sigma=72 \mathrm{mN} \mathrm{m}^{-1}$. Gravity is included as $g=9.81 \mathrm{~m} \mathrm{~s}^{-2}$. We do not take into account the different properties of the bottom liquid in the simulation, and therefore do not include any Marangoni or variable-density effects between the two liquids. More details about the adaptive grid refinement can be found in Popinet (2003) and Thoraval et al. (2012). 


\section{Results and discussion}

In what follows, we start by imaging water drops impacting on water pools. For Reynolds number up to $\sim 12000$, no bubble rings are entrapped, but unexpectedly we see an azimuthal undulation in the neck region, which breaks the axisymmetry. However, above this Reynolds number, a sequence of partial bubble rings are entrapped in the neck, which subsequently break into numerous micro-bubbles. These micro-bubbles display random sideways motions, suggesting underlying vortical structures.

Then we use water drops impacting on ethanol or methanol pools, which produces more regular bubble rings, and the difference in the index of refraction reveals intricate vortical tangles with both streamwise and azimuthal components.

We characterize the radial location where the first bubble rings are entrapped, as well as their radial spacing. We also show that the pool depth and drop shape at impact can alter the details, but not the qualitative dynamics of the bubble-ring entrapment.

Finally, we observe experimentally the dynamics of the entrapment mechanism that was described in the numerical simulations of Thoraval et al. (2012), as well as the three-dimensional effects that could not be captured by their axisymmetric simulations. Detailed images are presented, showing the rotation around the bubble rings, the splashing following ring entrapment, as well as the instabilities and entanglement of the vortices.

\subsection{Isolated bubbles and multiple bubble rings for a water pool}

We start by looking at the impact of the water drop on a water layer. Figure 4 shows the early evolution of the outer neck contact of the drop with the pool. The contracting inner air disk is visible on the left side of the images in figure $4(d, e)$. Note that we are only looking at the early contact when the neck has not reached the size of the drop, as shown in the sketch in figure $1(b)$. The radius of the neck in the last panel of figure $4(d)$ has only reached $37 \%$ of the drop radius.

Figure 4 shows that even for low impact velocities the neck region between the drop and the pool does not remain smooth and axisymmetric, but develops azimuthal undulations. For the lowest impact velocities these undulations have long wavelengths and do not entrap bubbles, see figure $4(a-c)$. However, with increased impact velocity $V$ the wavelength reduces and their amplitude grows more rapidly. In figure $4(d)$ these undulations appear first in the second panel and grow in amplitude during the radial motion, but individual bumps saturate and are often being pulled back by surface tension. The shapes are irregular, but we can glean a characteristic wavelength from the third panel in figure $4(d)$, giving $\lambda \sim 53 \mu \mathrm{m}$, corresponding to 73 undulations around the periphery. These undulations appear when the ejecta emerges, pulling local sheets of air under the ejecta on both or alternating sides of it. These local sheets can be pulled along with the ejecta base, with only occasional bubbles entrained, when these small azimuthal air discs make contact across the thin air layer, as is shown in a longer sequence of frames in figure 5. Individual bubble entrapments can also occur in the troughs between the undulations.

In figure 6 we show the growth of the maximum undulation amplitude, measured between the troughs and peaks, see inset in the figure. The growth rate slows down with radial distance. For reference we fit a viscous-type length scale,

$$
L_{v}=C \sqrt{v\left(t-t_{o}\right)}
$$


(a)

$(b)$

(c)

(d)
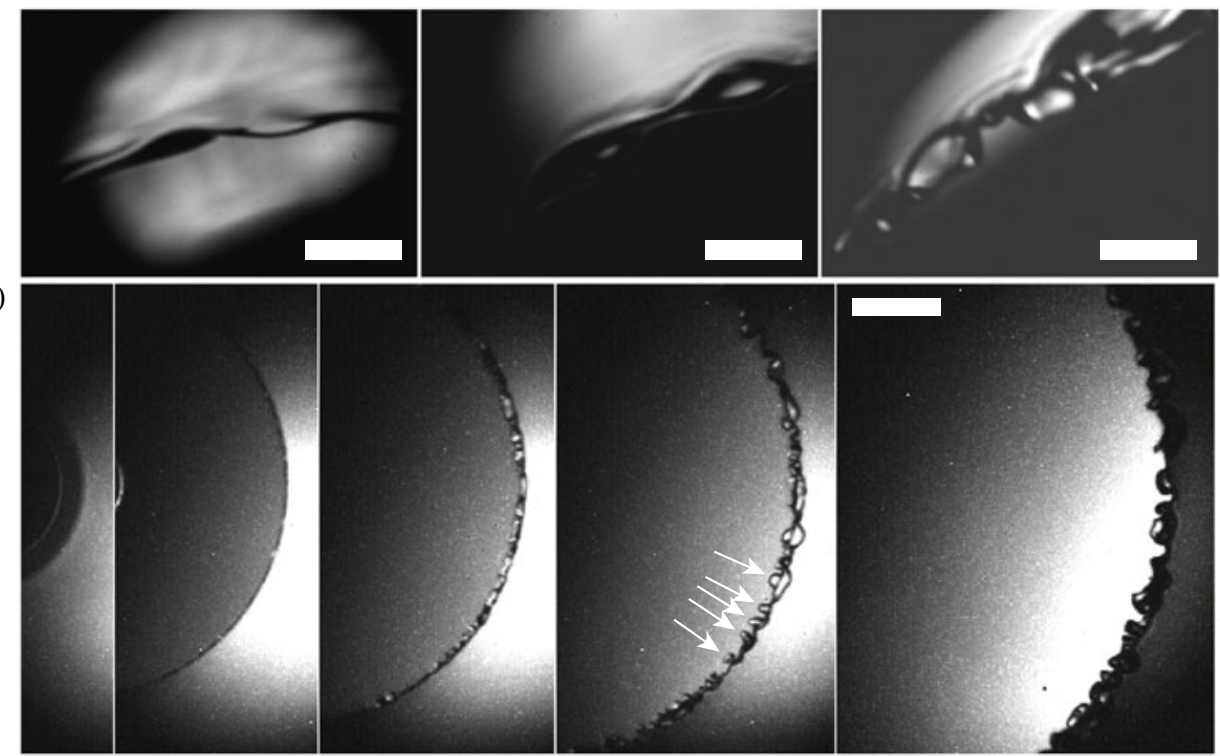

(e)

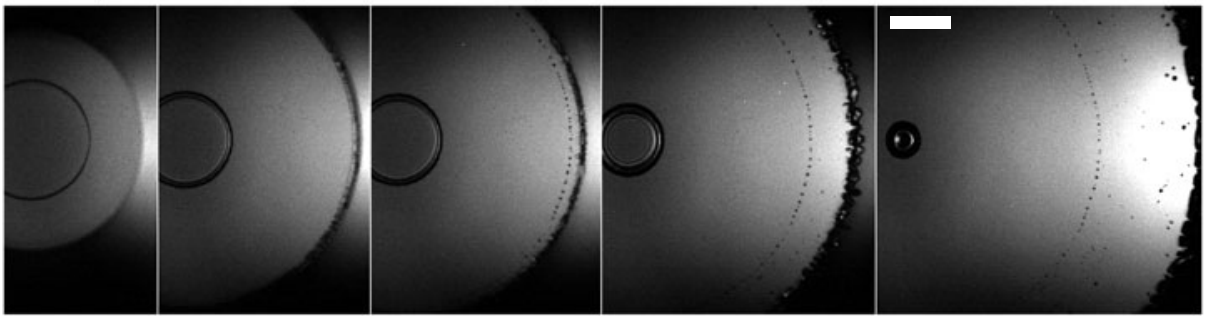

$(f)$

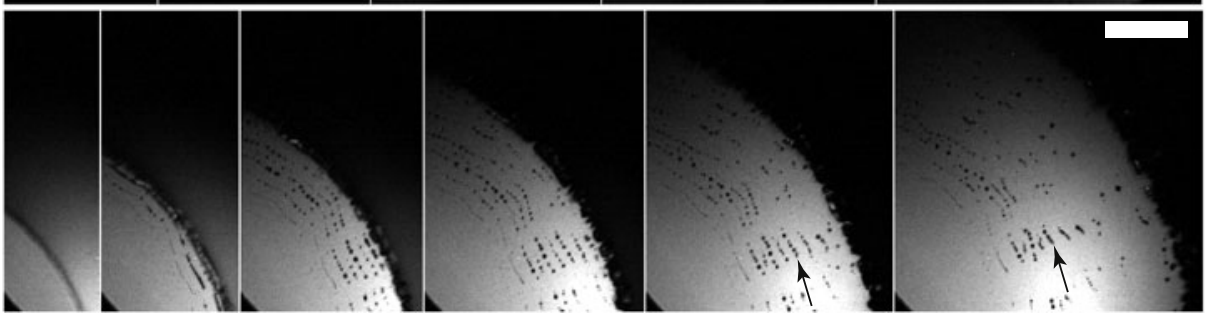

FIGURE 4. Early contact of a water drop impacting on a $\delta=250 \mu \mathrm{m}$ deep layer of water, observed from below, as described in figure 1. $(a-c)$ Neck between the drop and the pool. No bubble entrapment is observed at low impact velocities. An azimuthal instability develops on the ejecta, with a wavelength decreasing with increasing $\operatorname{Re}$. (a) $R e=3610, W e=39$, $K=2360, \alpha=0.94,(b) \operatorname{Re}=4400, W e=58, K=3860, \alpha=1.17,(c) \operatorname{Re}=5470, W e=90$, $K=6640, \alpha=0.86$. $(d)$ For an intermediate impact velocity, individual micro-bubbles can be entrapped. Frames are shown at 1, 13, 18, 25 and $46 \mu$ s after the first contact. $R e=11400$, $W e=394, K=42100, \alpha=1.05$. (e) For slightly higher impact velocity, the drop entraps one bubble ring and isolated bubbles, shown at $t=3,11,13,21$ and $40 \mu \mathrm{s}$. $R e=13300$, $W e=535, K=61800, \alpha=0.98$. $(f)$ Multiple bubble rings. Frames are shown at $\sim 5,9,13$, 17,21 and $32 \mu \mathrm{s}$ after first contact. $R e=12900, W e=506, K=57600, \alpha=0.80$. The scale bars are all $200 \mu \mathrm{m}$ long. See also supplementary movies, available at http://dx.doi.org/10. 1017/jfm.2013.147. 

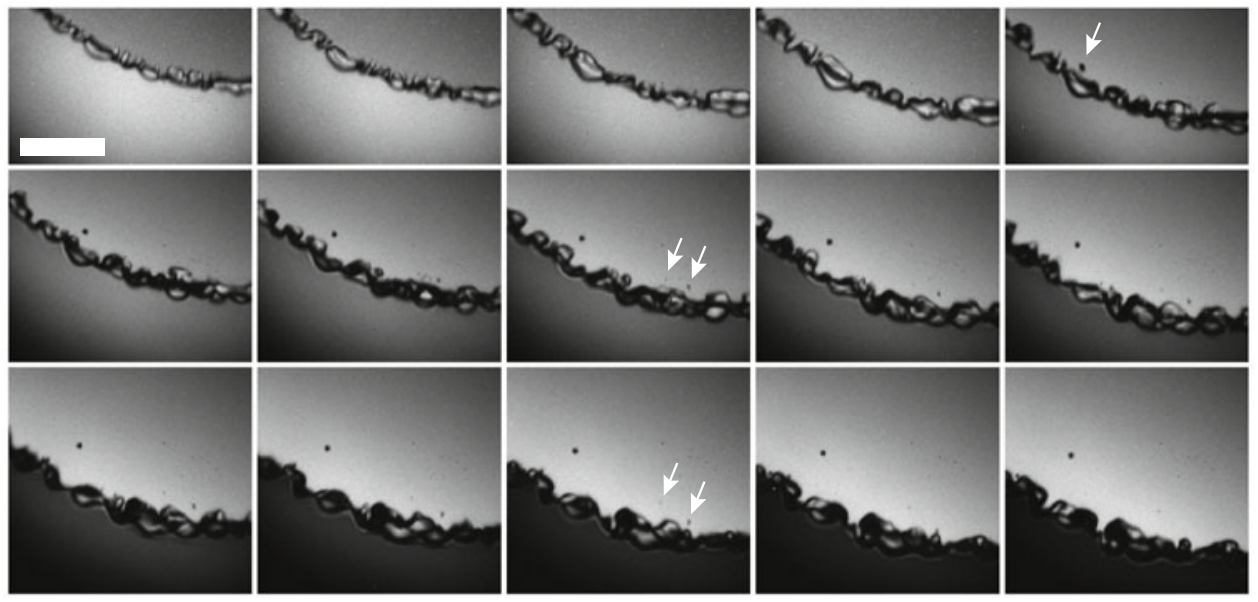

FIGURE 5. Details of individual micro-bubble entrapments for the same conditions as in figure $4(d)$. Frames are shown $2 \mu$ s apart. The top row shows the dynamics leading to the entrapment of $\mathrm{a} \simeq 10 \mu \mathrm{m}$ diameter bubble. On the second row, two smaller bubbles of diameter $\simeq 4$ and $6 \mu \mathrm{m}$ are separating from the right-hand part of the edge. While the first one stays behind the edge, and can be seen in the last frame, the second one is re-absorbed into the neck in the last row. The scale bar is $200 \mu \mathrm{m}$ long.

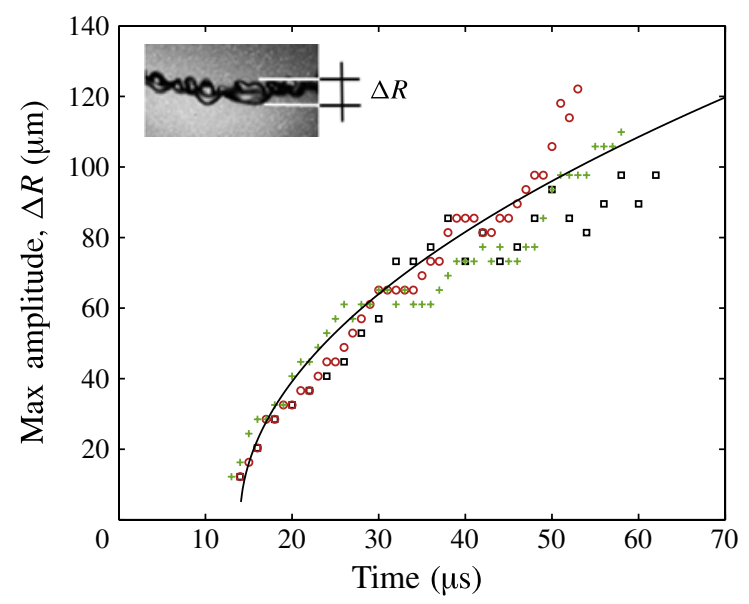

FIGURE 6. The maximum amplitude of the undulations of the front in the neck region, for water drop onto water pool. Data from three realizations, for the same conditions as figures $4(d)$ and 5. The solid line shows formula (3.1), with $C=14$. The inset shows how the amplitude is measured.

where $C=14$ and $t_{o}$ is the time of first observed undulations on the front. This suggests that the azimuthal wavelength is significantly longer than the thickness of the nascent ejecta, which should be of the order $\sqrt{v t}$, see Josserand \& Zaleski (2003). Based on this undulation length scale $\Delta R$, along with the neck velocity and water properties, the local $R e_{\Delta R} \sim 700$ and $W e_{\Delta R} \sim 100$, suggesting inertia, viscous forces and surface tension all play a role in the dynamics. 
Figure 5 shows that the characteristic azimuthal wavelength of the undulations also grows during the radial motion of the front, but this is more difficult to quantify.

It is curious that some air entrapment in breaking gravity waves has a superficially similar appearance (Kiger \& Duncan 2012, their figure 11), but is clearly driven by a different mechanism and is three orders of magnitude larger in size.

For slightly higher impact velocity, the entrapment of rather irregular bubble arcs begins. Figure $4(f)$ shows up to 10 such partial rings. The average radial spacing of the adjacent bubble rings is $\simeq 26 \mu \mathrm{m}$. The air cylinders then break up into a row of bubbles through surface-tension-driven Rayleigh instability. Bubbles are often shifted sideways in the azimuthal direction during the radial spreading (arrows in figure $4 f$ and see supplementary movies, available at http://dx.doi.org/10.1017/jfm.2013.147). For a stationary hollow cylinder of diameter $d_{b}$ in an inviscid liquid, the most unstable wavelength is $\lambda_{m}=\pi d_{b} / 0.484$. The characteristic time scale of the exponential growth $\sim \exp \left(t / \tau_{\sigma}\right)$ of the breakup is given by $\tau_{\sigma}=1.22 \sqrt{\rho r_{b}^{3} / \sigma}$ (Chandrasekhar 1961). The radii of the bubble arcs $r_{b}$ for a water layer, in figure $4(f)$, are $\sim 3 \mu \mathrm{m}$ and they break up in about $\sim 3 \mu \mathrm{s}$, which is $4 \tau_{\sigma}$.

Thereby, the first bubble-ring entrapment for water occurs around $R e \simeq 12000$ and $K \simeq 50000$. These values are consistent with the numerical results of Thoraval et al. (2012), where no bubble-ring entrapment is observed for $\operatorname{Re}=10000$ and $K=30000$, and a row of bubble rings is observed for $R e=14500$ and $K=74400$. In the former case, the ejecta sheet is thicker, because of the stronger surface tension effects on the ejecta sheet owing to the lower value of the splashing parameter $K$. It is re-absorbed in the drop or the pool during the oscillations, and no bubble-ring entrapment is predicted. However, in the latter case, at higher $K$, the ejecta sheet is thinner, and its oscillations entrap a row of bubble rings at the core of vortex rings when it impacts on and connects with the drop or the pool.

However, the comparison of figures $4(e)$ and $4(f)$ shows that more bubble rings can be observed at a slightly lower $R e$ and $K$. This suggests that the $R e$ of the impact is not enough to characterize the bubble-ring entrapment. We will show in $\$ 3.4$ the critical effect of the drop shape. Moreover, the azimuthal instabilities also affect the air entrapment, and individual bubble entrapments have been observed at slightly lower $R e$ in figures $4(d)$ and 5.

Note that in the work of Castrejón-Pita et al. (2012), the vortex street is observed for conditions similar to figure $4(f)$ of Thoraval et al. (2012), where no bubble-ring entrapment was predicted. Considering the large diameter of the drop they are using, it is not clear if the bubbles they observe are part of a bubble ring or isolated bubbles. They are also looking at a larger view and longer time evolution, that could be out of the field of view used in the current investigation (see perspective in figure 1). However, their alternating vortices are a new observation, clearly different from the isolated vortex rings produced by much lower impact velocities, see Peck \& Sigurdson (1994).

\subsection{Bubble rings for miscible liquids}

The bubble-ring entrapment becomes more regular for the impacts on ethanol and methanol pools (figure 7), perhaps due to the lower surface tension of these liquids (table 1). Contrary to the impacts on water films, no azimuthal instability develops on the neck of the ejecta, which remains perfectly smooth in figure $7(a-c)$. At higher impact velocities bubble rings are entrapped. Figure $7(d)$ shows at least 10 bubble rings. Many of them are entrapped axisymmetrically over the entire image view, which can span around $90^{\circ}$ angular sector. 
(a)

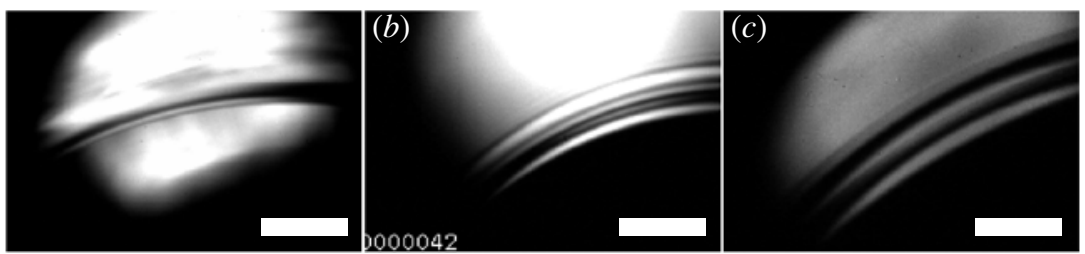

(d)

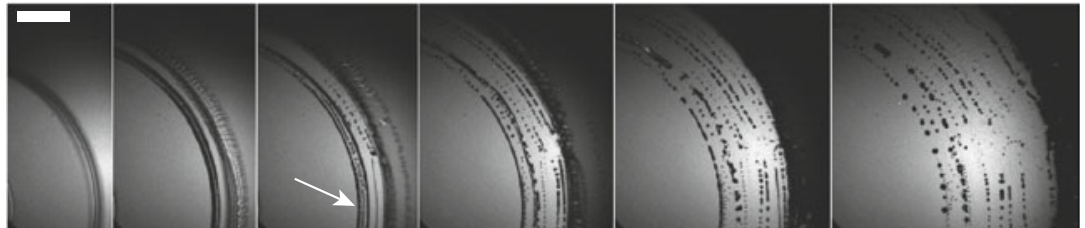

(e)

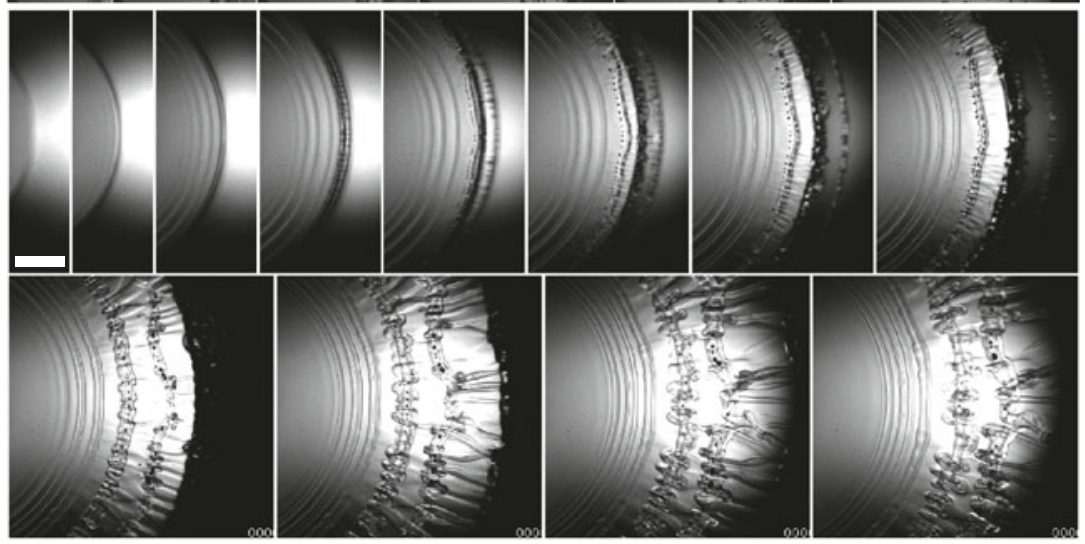

(f)

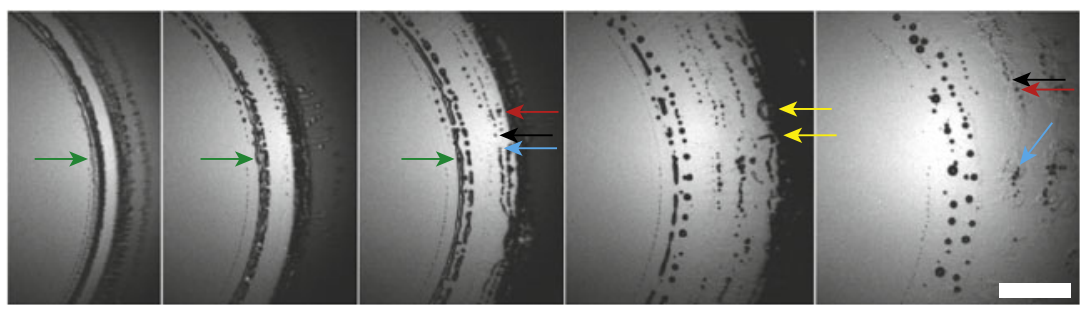

FIGURE 7. Early contact of a water drop impacting on a pool of a miscible liquid (ethanol or methanol), observed from below, as described in figure 1. $(a-c)$ Neck between the drop and the pool. No bubble entrapment is observed at low impact velocities on a thin film of ethanol $(\delta \simeq 250 \mu \mathrm{m})$. Unlike the water film, no azimuthal instability develops on the ejecta. (a) $R e=3610, \alpha=0.94$, (b) $R e=4400, \alpha=1.17,(c) \operatorname{Re}=5470, \alpha=0.86$. (d) Bubble rings for a water drop impacting on a methanol layer $(R e=12900, \alpha=0.80, \delta=50 \mu \mathrm{m})$. Frames are shown at 5, 9, 11, 15, 20 and $33 \mu$ s after first contact. (e) First oscillations of the ejecta sheet, followed by entrapment of bubble rings, for a film of ethanol $(\operatorname{Re}=13300, \alpha=0.98$, $\delta=250 \mu \mathrm{m})$. Azimuthal instabilities appear in the ejecta sheet, before its rim detaches in a liquid toroid. The first eight frames are shown $4 \mu$ s apart, and then $20 \mu s$. $(f)$ Detail of the bubble entrapment dynamics for the same conditions as in $(d)$. The first frame shows the entrapment of a superposition of air sheets, later breaking into patches and then into micro-bubbles (green arrows). Three bubbles are identified in the third and last frames by red, black and blue arrows, showing their strong sideways motion. Bubble arcs with legs in the radial direction are identified by the yellow arrows. Frames are shown 3, 6, 15 and $60 \mu$ s after the first one. The scale bars are all $200 \mu \mathrm{m}$ long. 
In some instances thin ribbons of air are entrapped and subsequently break up into sub-rings and thereafter bubble rings, as highlighted by an arrow in figure $7(d)$. The frames in figure $7(f)$ detail a similar sequence of air entrapments. One air patch is identified by the green arrow in the second frame, superposed with another air sheet behind it. In the third frame, the air patch breaks into a bubble (identified by the green arrow), and a small air cylinder at a larger radial location, while the air sheet behind contracts into a cylinder. The vertical superposition of the air entrapments is clearly shown by the fact that the air bubble is observed at the same radial location as the air cylinder. This supports the mechanism that air can be entrapped both above and below the ejecta sheet, as was shown in the numerical simulations of Thoraval et al. (2012).

In a similar way as for water pools, figure $7(f)$ also demonstrates strong sideways motions of bubbles. Three bubbles are identified in the third frame by red, black and blue arrows. Their corresponding location is marked by the same coloured arrows in the last frame. Comparison between the black and red arrows shows that this sideways motion can be of different strength for adjacent rings. This bubble motion in the azimuthal direction results in their clustering at isolated locations.

Figure 8 shows a wider view of an impact taken at a lower frame rate of 10000 f.p.s. but larger pixel area of $896 \times 848$ px, using the Photron SA-5 CMOS camera. Each frame is frozen with a $1 \mu$ s exposure. The $100 \mu \mathrm{s}$ interframe time only shows us snapshots of the phenomenon, putting the earlier figures in perspective, with most of the earlier sequences occurring before the first image. In the second frame a multitude of splashed droplets appears from underneath the shadow of the drop, with some droplets planing on the pool surface, leaving behind narrow capillary wedges. Figure $8(a)$ shows a smooth central region, followed by a convoluted interface, suggesting stirring by the three-dimensional vortical structures (see §3.7). Similar stirring can be inferred from the side shadowgraph imaging in Castrejón-Pita et al. (2012) (their figure 4). Figure $8(d)$ shows numerous isolated bubbles which have been redistributed by the vortical motions. The bubbles are mostly concentrated within the mushroom-like remnants of the vortical structures.

\subsection{First onset, number and spacing of bubble rings}

The first contact entraps a central air disk and forms a rapidly expanding outer liquid edge. Bubble rings are then formed as observed above. Figure 9(a) looks at the radial location where the first bubble ring is entrapped. We normalize the radius of the first ring $R_{1}$ with the horizontal drop radius when it first contacts the pool, $R_{h}=D_{h} / 2$. The data show a large spread, but an overall trend is for the onset to occur earlier for larger impact $R e$. The smallest entrapment radius is 0.18 , similar to the 0.2 limit observed by Thoroddsen (2002) for the onset of the ejecta sheet. This onset radius of the ejecta is also in agreement with the inviscid numerics of Weiss \& Yarin (1999).

Figure $9(b)$ shows the distance between adjacent bubble rings measured for numerous identical impact conditions. The spacing of the rings tends to increase with distance and the entrapped bubbles become larger.

\subsection{Effect of pool depth and drop shape}

To ascertain the influence of the pool depth we systematically vary the layer thickness $\delta$, from $\sim 25 \mu \mathrm{m}$ to $1 \mathrm{~mm}$. Figure $10(a-c)$ compares the bubble rings for the three smallest pool depths $\delta$. The ring structures are qualitatively similar in all cases, but the shallowest pool shows the earliest and finest bubble rings, some of which are sub-pixel in diameter. The second ring in figure $10(a)$ allows us to measure the separation of micro-bubbles, giving $\lambda=8.8 \mu \mathrm{m}$, suggesting a diameter of the original 
(a)

(c)
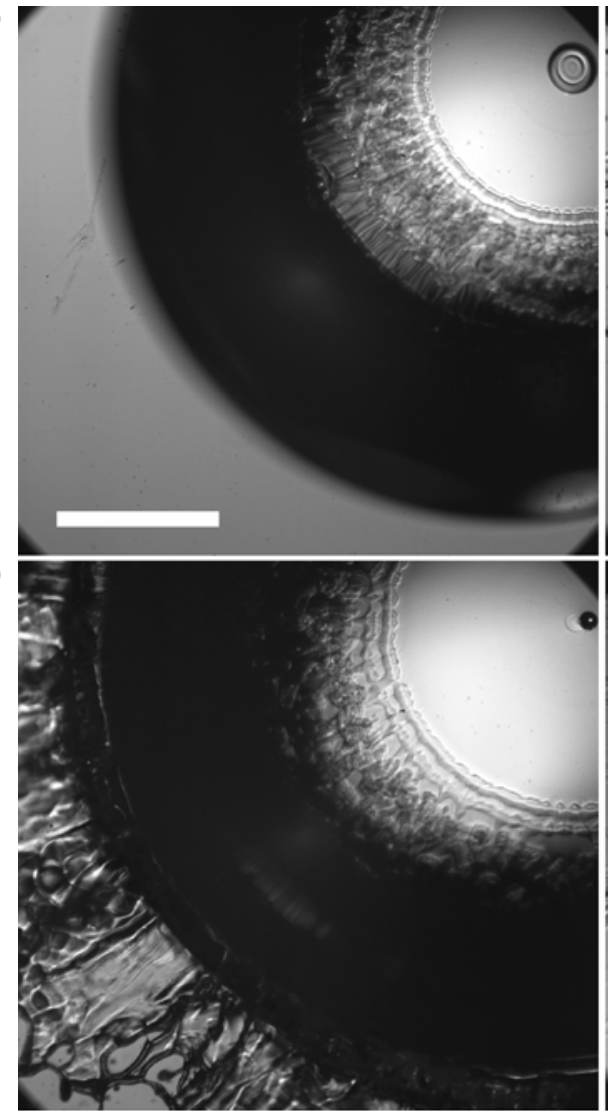

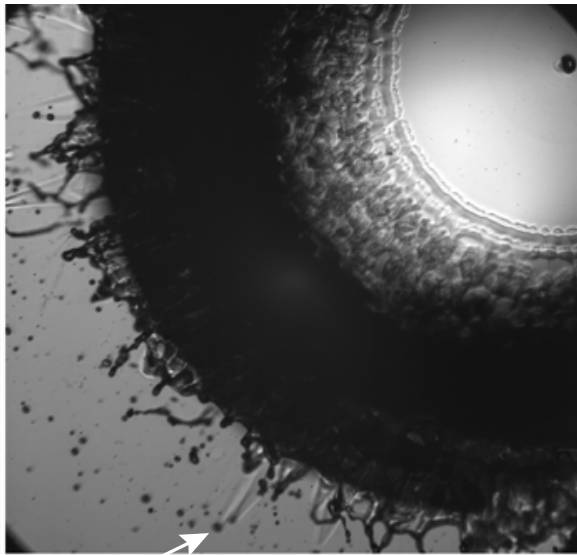

(b)

FIGURE 8. Larger view of a water drop impacting on ethanol, at times $t \sim(a) 50$, (b) 150 , (c) 250 and $(d) 1150 \mu \mathrm{s}$ after first contact $(R e=14500, \alpha=0.94, \delta \simeq 125 \mu \mathrm{m})$. Frames taken from videos using Photron SA-5 at 10000 f.p.s. with a $1 \mu$ s exposure time. The arrow at the bottom of the second frame points at two splashed droplets planing on the surface, as shown by the capillary wedges left behind them. The central bubble has drifted out of the image in the last frame. The scale bar is $1 \mathrm{~mm}$.

air torus $d_{\text {tor }} \simeq 1.4 \mu \mathrm{m}$. The earliest ring appears even smaller, arrow in figure $10(a)$. Figure $10(d)$ shows fewer but qualitatively similar ring entrapment, for much thicker layer.

Previous results of Thoraval et al. (2012) suggested that the oscillations of the base of the ejecta sheet are responsible for the bubble-ring entrapment, for a drop impacting on a deep pool at high $R e$ and $K$. Figure 11 shows numerical results for three different shallower pool depths. The two-liquid interface is highlighted by colouring the drop and pool differently. It shows that the same mechanism is also present for shallow pools, with only minor changes. However, the drop penetrates further into the pool for larger $\delta$. The vortex street is therefore constrained in a shallower region for shallower pools, and develops more horizontally. This constraining effect increases the maximum liquid velocity by as much as $20 \%$, as shown in figure 12(a). The boundary layer developing on the glass plate could be important for the shallowest pools.

The first bubble ring is entrapped at $R_{1} / R$ of respectively $0.37,0.31$ and 0.28 for pool depths $\delta=800,200$ and $100 \mu \mathrm{m}$ respectively. This confirms the previous 
246

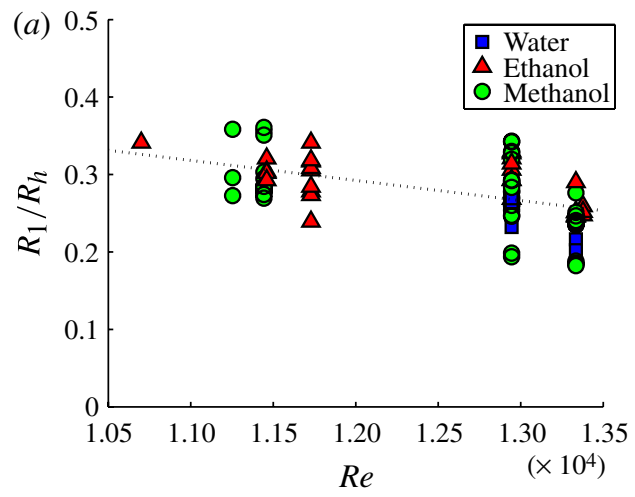

M.-J. Thoraval, K. Takehara, T. G. Etoh and S. T. Thoroddsen

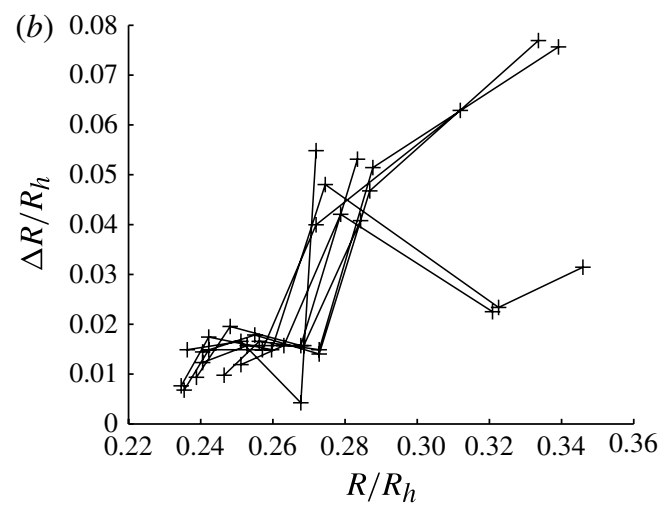

FIGURE 9. (a) Radial location of first onset of bubble rings for a water drop impacting on a water, ethanol or methanol layer of different depths (see also figure 12b). (b) Spacing of adjacent rings $\Delta R$, versus radial distance $R$ from the impact centre. Data from eight different realizations for a methanol pool $(R e=13300, \alpha=0.98, \delta=250 \mu \mathrm{m})$.

(a)

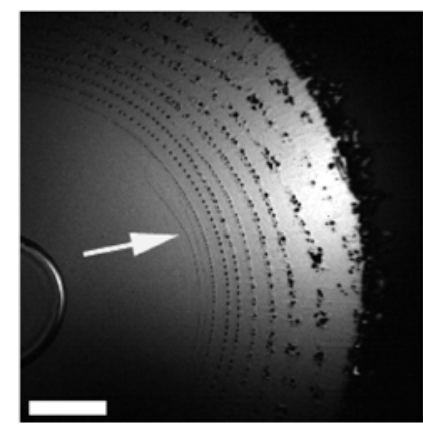

(b)

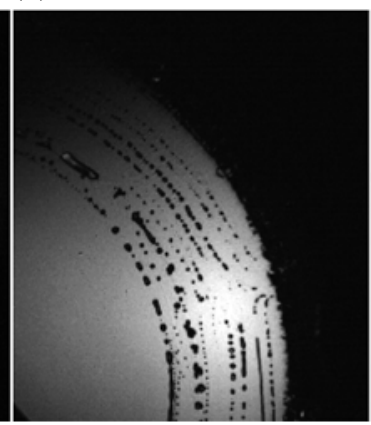

(c)

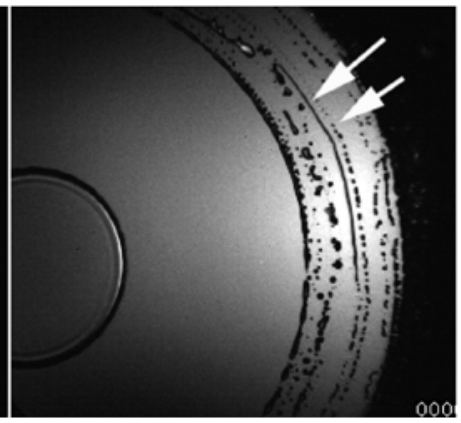

(d)

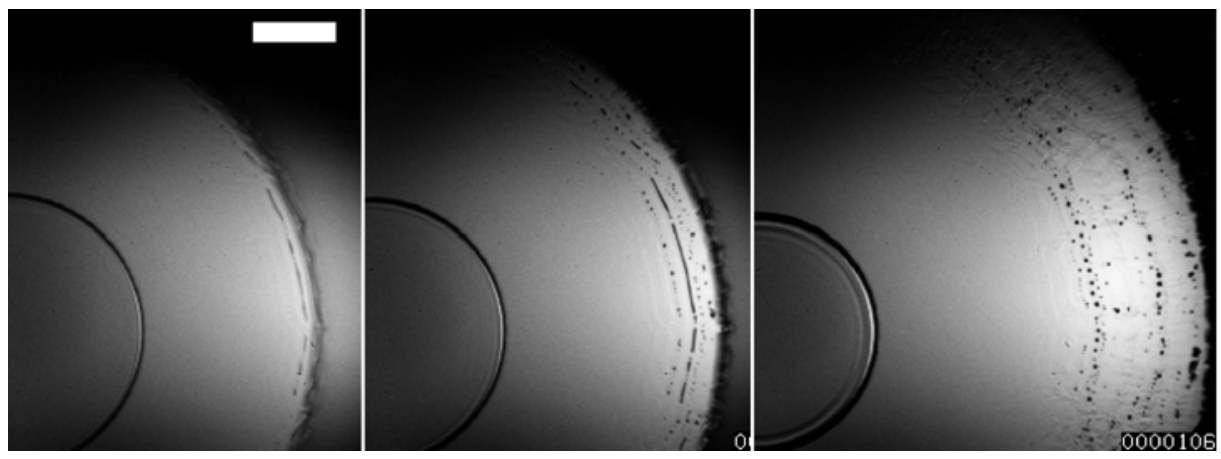

FIGURE 10. Effect of pool depth on the bubble rings for a water drop impacting on methanol, with $\delta \simeq 25(a), 50(b)$ and $75 \mu \mathrm{m}(c)(R e=12900, \alpha=0.80)$. The frames are all shown $24 \mu$ s after the first contact in $(a-c)$. $(d)$ Deeper methanol pool with $\delta \simeq 500 \mu \mathrm{m}$. Frames shown at 10, 14 and $28 \mu$ s after first contact. The scale bars are $200 \mu \mathrm{m}$ long. See also supplementary movies.

experimental observation of figure 10 that earlier rings are observed for shallower pools. Figure $12(b)$ also shows this experimentally in a more systematic way, but the difference is not very pronounced. The radial location of this first entrapment 
(a)

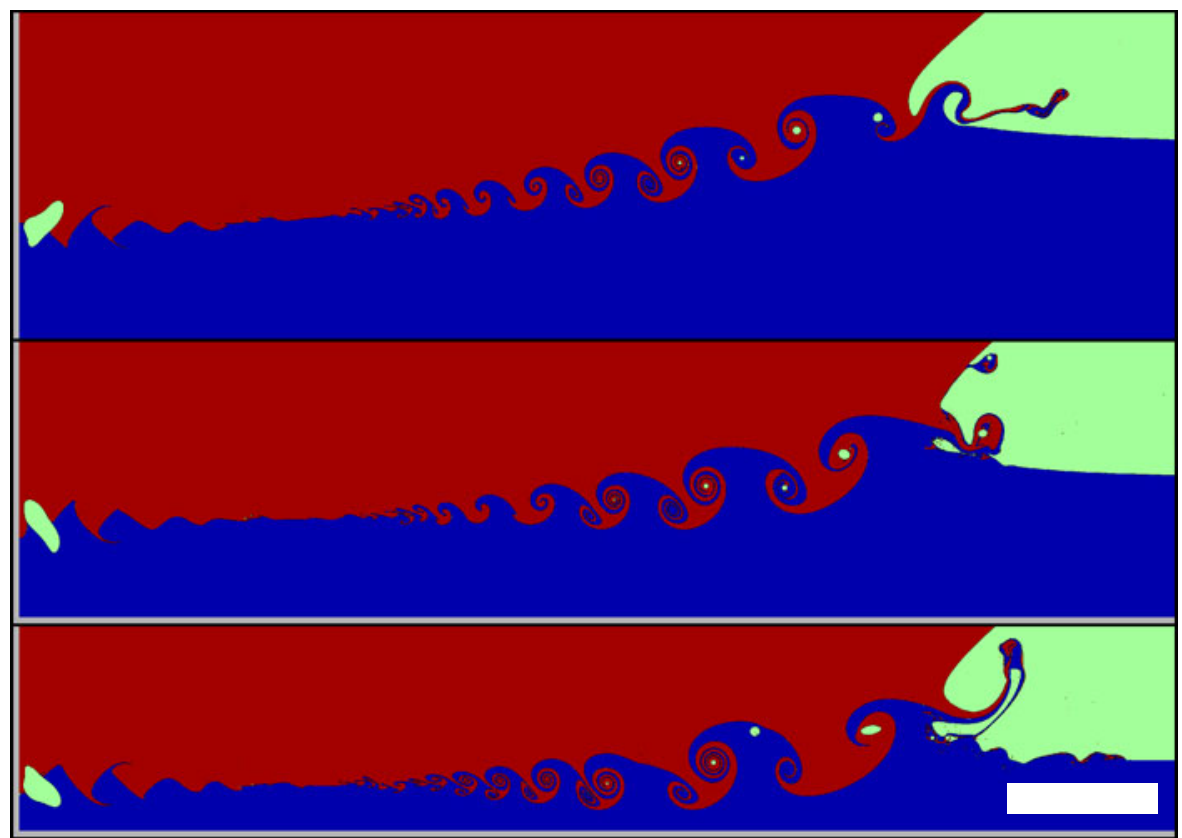

FIGURE 11. Numerical results for the effect of pool depth on the vortex street at $t^{*}=0.0526$, for $R e=13700$. Pool depth $\delta=800 \mu \mathrm{m}(a), 200 \mu \mathrm{m}(b), 100 \mu \mathrm{m}(c)$. The top of the 3 images are at the same location, relative to the original pool surface. The pool depth is larger than shown in the image in $(a)$, but is completely included for $(b, c)$, where the bottom is indicated in grey. The maximum and minimum level of refinement in the domain are respectively 12500 and 778 cells per drop diameter and $D_{\text {cut }}=1.3 \mu \mathrm{m}$. The bar is $200 \mu \mathrm{m}$ long. We can observe that one part of the ejecta sheet is climbing on the drop in $(b)$, while the main ejecta sheet continues to emerge below. This is similar to what was observed numerically by Thoraval et al. (2012) and experimentally by Zhang et al. (2012). The formation of this higher part of the ejecta sheet can be observed in the supplementary movie. It then merges with the drop in this case.

is also consistent between experimental and numerical observations, even though the numerical simulations only consider one liquid.

We observe experimentally that the most robust bubble rings are produced by a flat-bottom drop, as shown by the comparison of figures $4(e)$ and $4(f)$ and figures $7(d)$ and 7(e), with fewest bubble rings in figure 7(e), which is more spherical. The largest number of rings is also produced by such oblate drops (figures $4 f$ and $20 b$ ). This is consistent with the numerical results of figure 13, showing a larger number of rings for the oblate drop. They even suggest that a prolate drop could completely suppress the bubble-ring entrapments for the same effective diameter, as is shown in figure 13(a).

\subsection{Edge breakup and splashing}

Numerical simulations have shown that the ejecta sheet can impact alternately on the drop and the pool during the vortex shedding. The tip of the ejecta can thus detach into a liquid torus exiting the neck region at high speed (Thoraval et al. 2012). Such tori are highly unstable to Rayleigh instability and break rapidly into splashed micro-droplets of similar sizes. However, this earliest splashing of micro- 

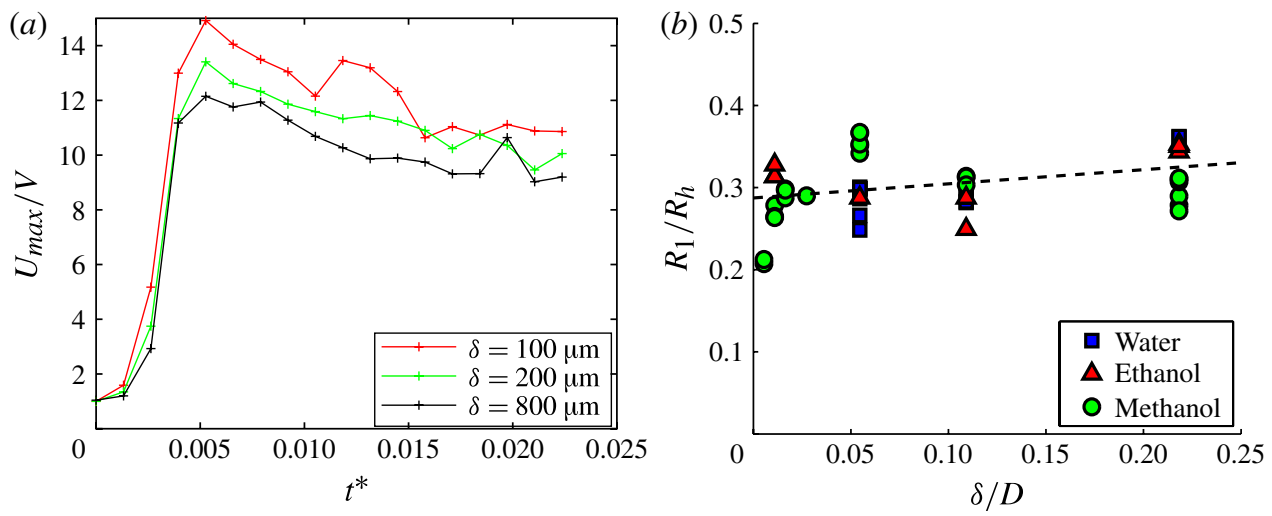

FIgURE 12. (a) Maximum velocity $U_{\max }$ in the liquid from the numerical simulations in figure 11, for three different pool depths. (b) Experimental observation of the first bubble ring entrapment radius $(R e=12900, \alpha=0.80)$ for different pool depths.

(a)
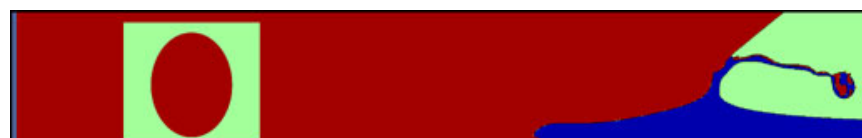

(b)

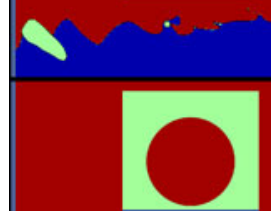

(c)
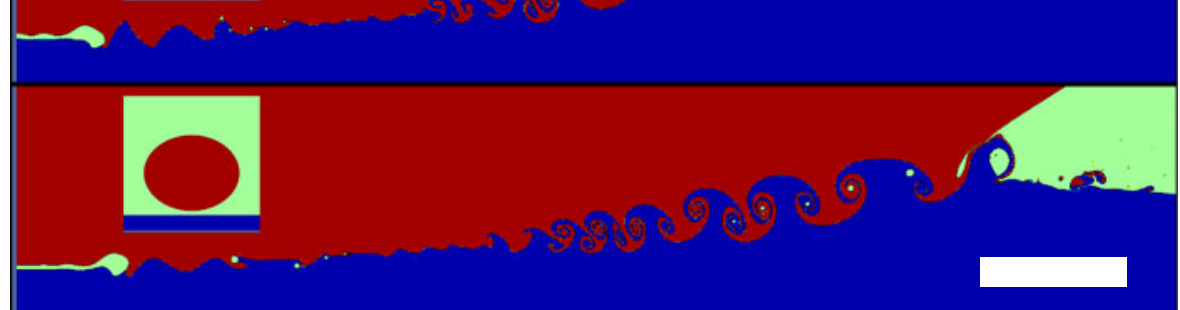

FIGURE 13. Effect of drop shape on the early dynamics at $t^{*}=0.0406$, for $R e=12900$ and $\delta=800 \mu \mathrm{m}$. The drop is modelled as an ellipsoid of revolution, where $\alpha$ is $(a) 1.29,(b) 1$, (c) 0.79. $(a, c)$ Correspond to the maximum horizontal deformations of the fitting equation (2.4), with $a=0.162$, keeping the same effective diameter. The maximum and minimum level of refinement in the domain are respectively 5240 and 655 cells per drop diameter, and $D_{\text {cut }}=3.1 \mu \mathrm{m}$. The scale bar is $200 \mu \mathrm{m}$ long. See also supplementary movies.

droplets by axisymmetric breakup of the ejecta sheet had not been observed previously in experiments.

By looking carefully at figures $7(d-f)$ and 8 , we can identify this early splashing by the breakup of the tip of the ejecta sheet after the entrapment of a few bubble rings, as was suggested by the numerical simulations. The liquid toroid in figure $7(e)$ separates at $t^{*} \simeq 25 \mu \mathrm{s}$ after the first contact, with a velocity of $20.5 \mathrm{~m} \mathrm{~s}^{-1}$, which 


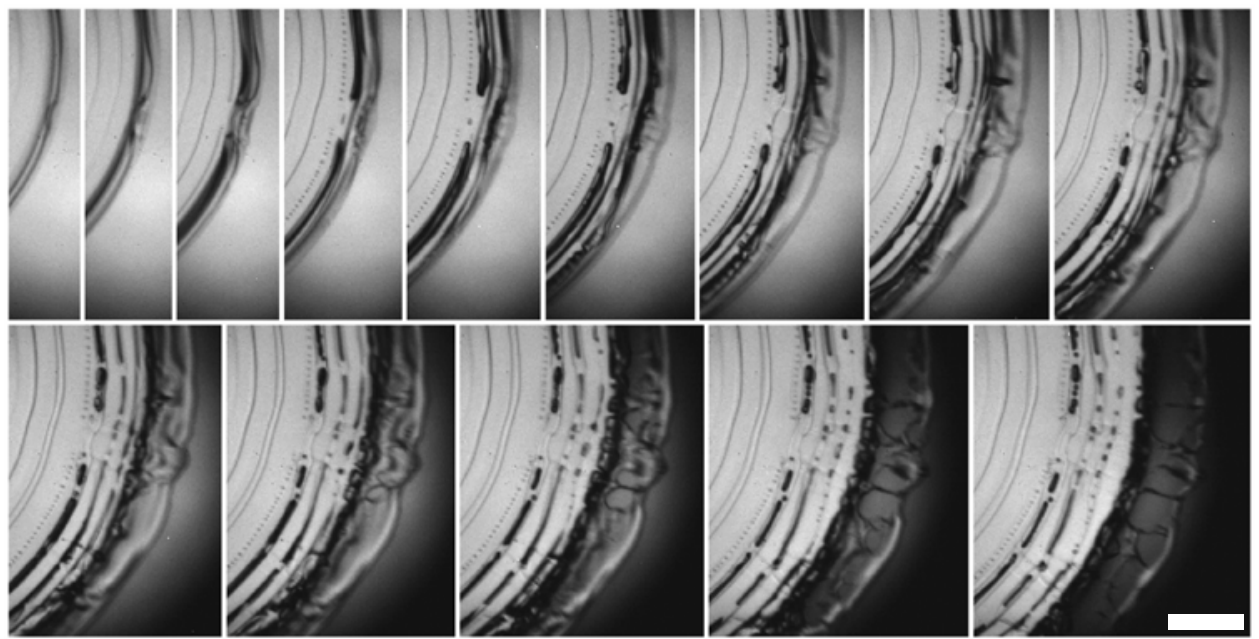

FIGURE 14. Details of the ejecta sheet breakup, for a water drop impacting on a thin film of ethanol $(R e=11700, \alpha=0.79, \delta=75 \mu \mathrm{m})$. The ejecta sheet starts to puncture in the second frame of the second row. The growth of the holes leaves tendrils connecting the neck to the liquid rim. The scale bar is $200 \mu \mathrm{m}$ long. Frames are shown $2 \mu \mathrm{s}$ apart.

corresponds to 7.1 times the impact velocity. The tip velocity of the ejecta sheet in the numerical simulations at the same non-dimensional time is 6.2 for $R e=12900$ (figure 13b), and 7.4 for $R e=13700$ (figure 11b), which is in excellent agreement with the experimental observations.

Figure 14 shows more detail of the breakup of the edge at a slightly lower $R e$. The ejecta sheet breaks via holes puncturing behind the rim. The thicker ejecta rim is therefore left connected to the neck by liquid tendrils, but subsequently becomes fully detached.

To remove the ambiguity of the bottom view, we have also looked at this early splashing from the side above the free surface. Figure 15(a) confirms the bottom view images, showing the ejecta sheet emerging from the neck, puncturing behind the rim and separating a liquid toroid from the neck. Liquid tendrils are also observed in the fourth frame, and are slingshot ahead of the rim, creating the protrusions observed in the last frame, similar to the last frame of the first row of figure 7(e). The slingshot of the broken ejecta sheet can also be observed in numerical simulation, as in the supplementary movie of figure 13(a), and is similar to the slingshot mechanism described in Thoroddsen et al. (2011).

Two consecutive liquid rings are observed in figure 15(b). It also shows the emergence of a greatly disturbed ejecta sheet after this early splashing. A larger bottom view confirms this side view observations in figure 15(c). This mechanism, of a detachment of a thin torus of liquid, explains the synchronized emergence of uniform-sized micro-droplets observed ahead of the main irregular ejecta sheet, see figure $15(d)$ as well as figure $2(c)$ in Thoroddsen (2002). The irregular sheet is clearly shown in figure 8 .

The splashing of several liquid tori is consistent with numerical simulations showing that the ejecta sheet can break up during the successive impacts on the drop and the pool. The supplementary movie of figure 13(a) shows such a case where the ejecta 


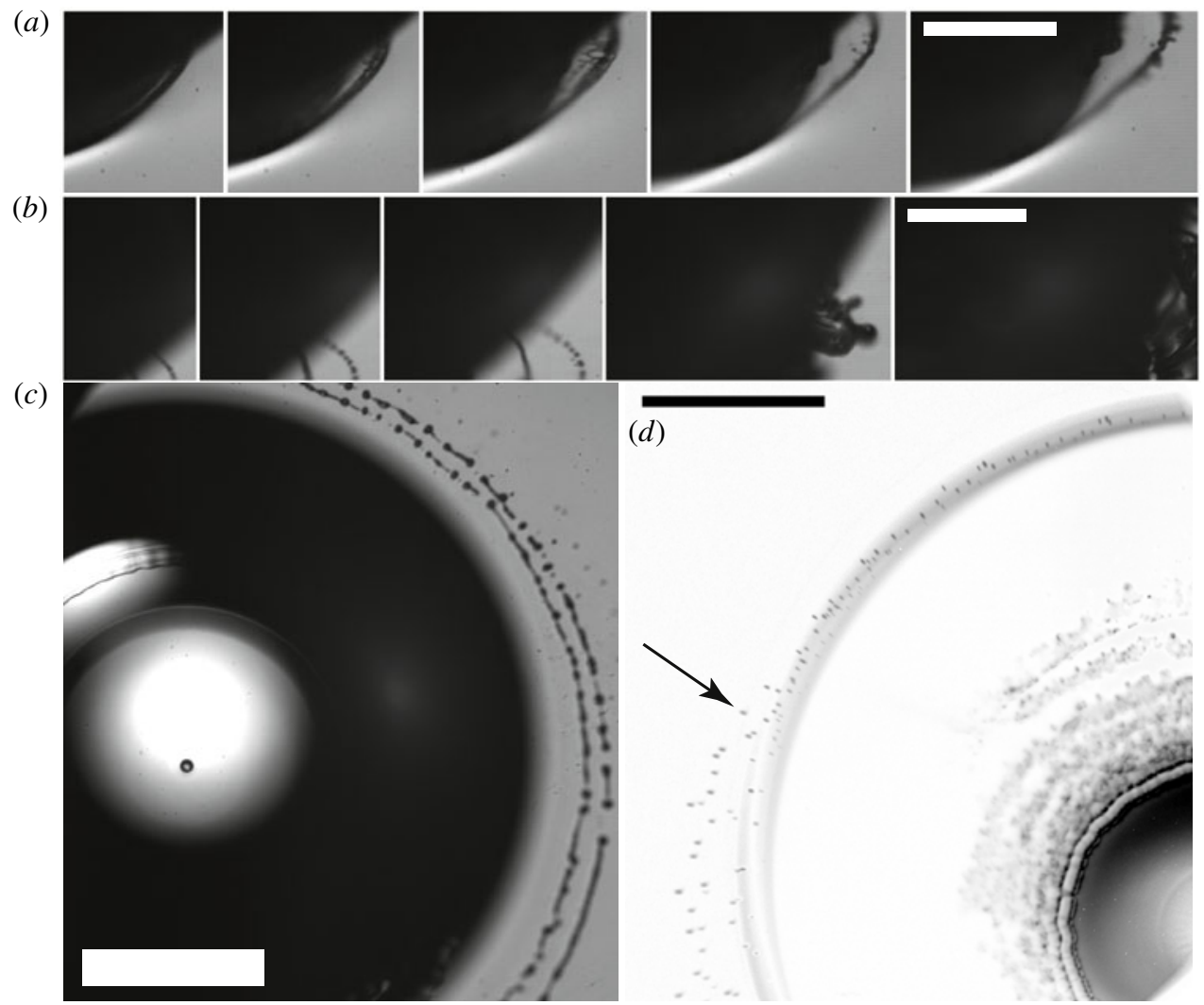

FIGURE 15. Detachment of liquid toroids by breakup of the ejecta sheet. Scale bars are $500 \mu \mathrm{m}$ for $(a, b)$ and $1 \mathrm{~mm}$ for $(c, d)$. All images have an exposure time of $1 \mu \mathrm{s}$. $(a)$ Side view of the liquid toroid detachment for a thin film of ethanol $(R e=12600, \alpha=1.11$, $\delta=125 \mu \mathrm{m})$. The ejecta sheet punctures in the third frame, breaking into tendrils. Those tendrils are slingshot in front of the liquid rim which has not yet broken, as observed in the last frame. The frames are shown $10 \mu$ s apart. $(b)$ Side view of two liquid toroid detachments for a thin film of methanol $(\operatorname{Re}=12600, \alpha=1.11, \delta=125 \mu \mathrm{m})$. The frames are shown $10 \mu \mathrm{s}$ apart for the first three frames, and then $80 \mu \mathrm{s}$. The splashing of liquid toroids is followed by the emergence of an irregular ejecta sheet, as can be seen also on the larger bottom view of figure 8. (c) Larger bottom view of two consecutive rings of liquid droplets and toroidal sections detaching for an ethanol pool $(\operatorname{Re}=11600, \alpha=0.86, \delta=125 \mu \mathrm{m})$. (d) First droplets emerging from underneath the drop in the same conditions as figure 8 . Image difference between two adjacent frames, to highlight the splashing droplets. Shown in inverted grey-scale with an exposure time of $1 \mu \mathrm{s}$.

sheet breaks first by climbing on the drop and then impacting on the pool, thus creating two consecutive liquid tori.

After the first bubble-ring entrapment, regular spanwise instabilities can appear in the ejecta sheet, as is clearly seen in the second panel of figure $7(d)$, as well as figure $7(e, f)$. The fine azimuthal breakup when the ejecta bends and impacts on a pool has been reported by Thoroddsen et al. (2011) (their figure 5) and may be of similar origin. Furthermore, the early appearance of similar azimuthal instabilities has also been observed by Thoroddsen et al. (2012) in a free-surface cusp, which is formed during a drop impacting on a solid surface. Numerical simulations show that the ejecta 


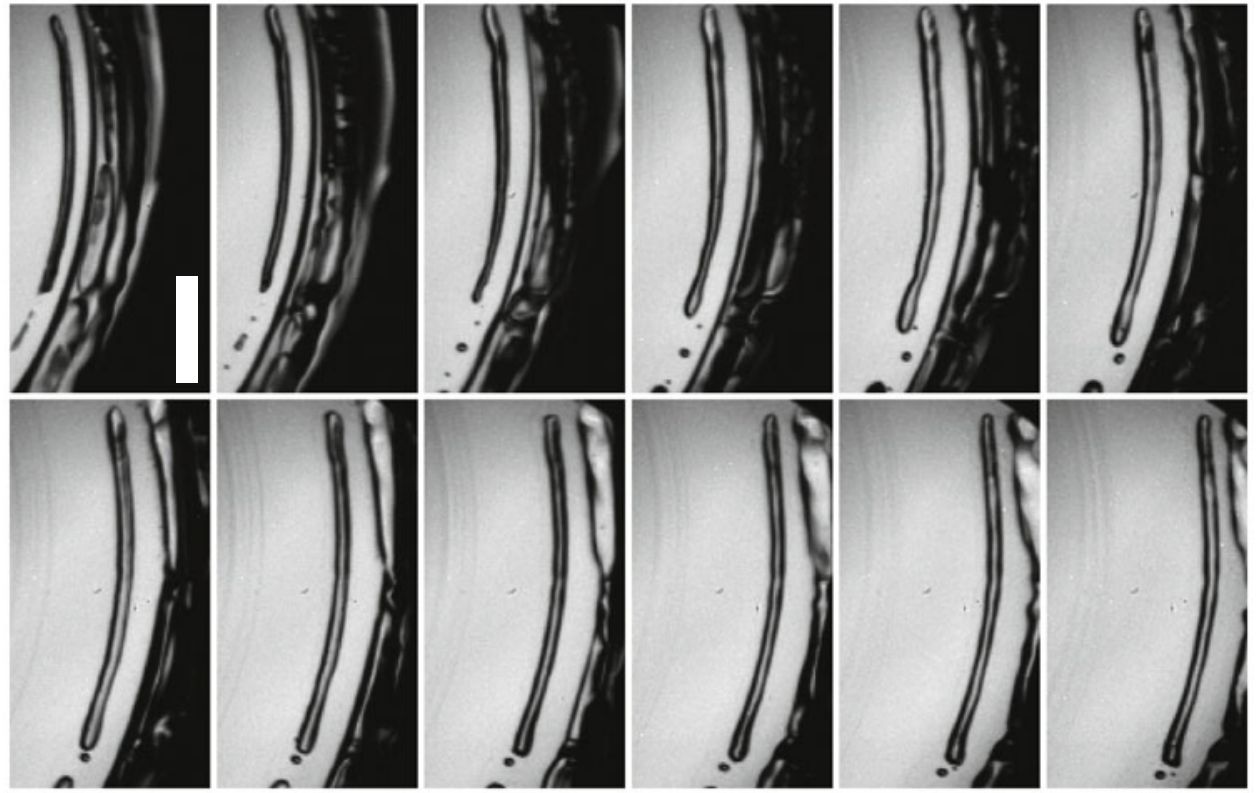

FIGURE 16. Entrapment of a large air cylinder for a water drop impacting on a thin film of methanol $(\operatorname{Re}=11400, \alpha=1.05, \delta=50 \mu \mathrm{m})$. The small bubble at the bottom right of the long cylinder makes a complete rotation around the cylinder between frames 4 and 12. The frames are shown $4 \mu \mathrm{s}$ apart. The scale bar is $200 \mu \mathrm{m}$ long.

sheet breaks when it impacts on the drop or the pool, by stretching between the new connection and the faster rim. This instability is therefore consistent with the impact of the ejecta sheet on the drop or the pool. Similar breakup of a liquid sheet by stretching was also observed experimentally by Roisman et al. (2007) for spray impacts.

\subsection{Vortex shedding and rotation around bubble rings}

The difference in refractive index between the drop and the pool (see table 1) allows us to visualize vorticity structures inside the liquid. As the coherent vortices bend and wrap up the interface between the two liquids, a dark line can be observed at their edges with our back-light imaging setup.

Numerical simulations have shown that the first oscillations of the base of the ejecta sheet have a smaller amplitude and do not entrap any bubble rings, see Thoraval et al. (2012) (their figure $4 c, f, g$ ) and our figures 11 and 13. This is consistent with our experimental observations of figures 7(e), 14, 18(a), 20, where dark arcs form before the first bubble rings. They show the shedding of vortices from the neck before the start of the bubble-ring entrapment.

As the neck moves outwards radially, the angle between the pool and the drop becomes larger, and bubble rings are entrapped, as observed above. Numerical simulations have shown that these rings are often entrapped in an alternating way at the top and the bottom of the ejecta sheet. At the same time, they shed vortices of alternating sign in the liquid. Bubble rings are therefore entrapped at the core of vortex rings. Dark lines are indeed observed experimentally around the bubbles, supporting this vortex-shedding scenario. The rotation is also made apparent by the dynamics of a micro-bubble rotating around a larger bubble cylinder in figure 16 . We can obtain an 


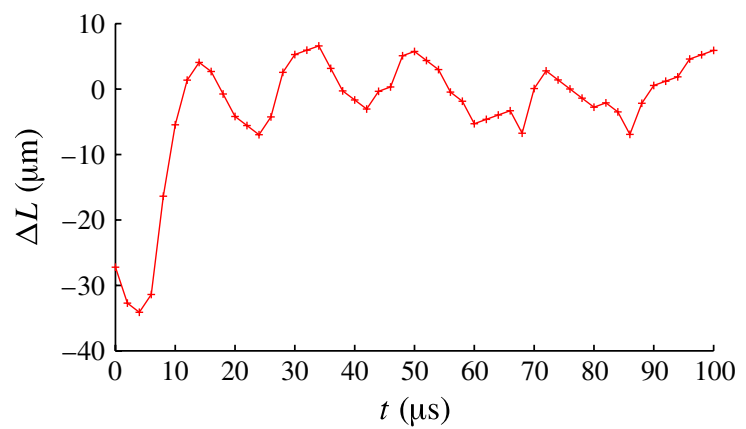

FIGURE 17. (Colour online) Tracking of a $2 \mu \mathrm{m}$ particle rotating around a vortex core for a drop impacting on a pool of ethanol $(\operatorname{Re}=11400, \alpha=1.05, \delta=125 \mu \mathrm{m}) . \Delta L$ is the distance from the initial position, minus the mean radial translation component identified by a linear regression. The period of rotation is here $\sim 18 \mu$ s.

estimate of the rotation speed in the vortex rings by tracking a $2 \mu \mathrm{m}$ particle seeded into the pool liquid (figure 17), giving a rotation period of $18 \mu$ s in this example.

The dynamics of the air entrapped in this vortex street is also affected by the rotation around it. One can expect the rotation to delay the capillary breakup of the air cylinders (Rosenthal 1962; Ashmore \& Stone 2004; Eggers \& Villermaux 2008). This is indeed evident in figure 16 , where the cylinder of diameter $\sim 29 \mu \mathrm{m}$ can be observed for more than $94 \mu$ s before breaking, corresponding to $t / \tau_{\sigma} \simeq 7$ based on the methanol properties. The air cylinder also elongates by $\sim 25 \%$ between the first and the last frame, consistent with the theory that it resides inside a vortex. The relative motion of the bottom tip of the air cylinder and the closest micro-bubble below shows that the stretching due to the radial motion cannot account for this elongation. Even smaller cylinders can be stabilized, as observed in figure 10(c). Two air tori are formed next to each other, with similar diameters $d_{t o r} \simeq 8 \mu \mathrm{m}$ (see arrows in the figure), but break up at very different times from formation, of $t / \tau_{\sigma} \simeq 4$ and 12 , based on the liquid properties of the methanol. These delayed breakups show the strong stabilization effect of the circulation around these air tubes. The breakup wavelength is also larger, as the theory suggests (Ashmore \& Stone 2004).

The row of vortices shed in the liquid can also interact with adjacent ones. In some realizations two closely entrapped air tori rotate around each other, with the line of small bubbles rotating around the bigger one. Figure 18(b) shows such a sequence, where we track one rotation, which takes $104 \mu$ s. Numerical simulations show that vortices of different strength are created at the top and bottom of the ejecta sheet (Thoraval et al. 2012). The longer time evolution shows that this difference can make the bubble rings rotate around each other while translating (figure $18 c$ and supplementary movies). These dynamics are consistent with the experimental observation of the rotation around bubble rings.

Some of the bubble tracks simply translate past each other during their radial motions, for example visible in the movies accompanying figures 18 and 20 . The bubbles are initially sitting at slightly different depths, and this difference in vertical location is amplified by the vorticity, as shown above. Moreover, as the bubble tori break into bubbles, their vertical width will slightly increase, sampling larger mean shear. These relative translations of bubbles could therefore result from a vertical mean gradient of horizontal velocity within the pool depth. 
(a)
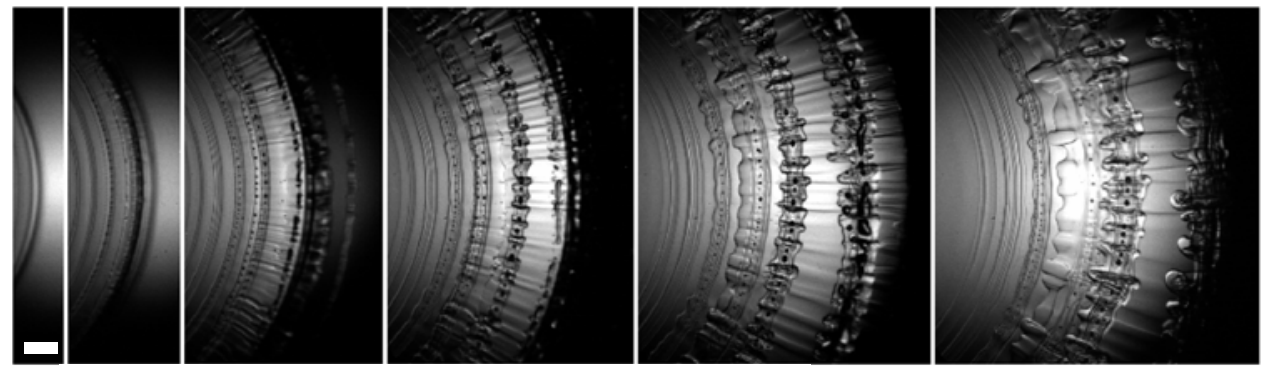

(b)
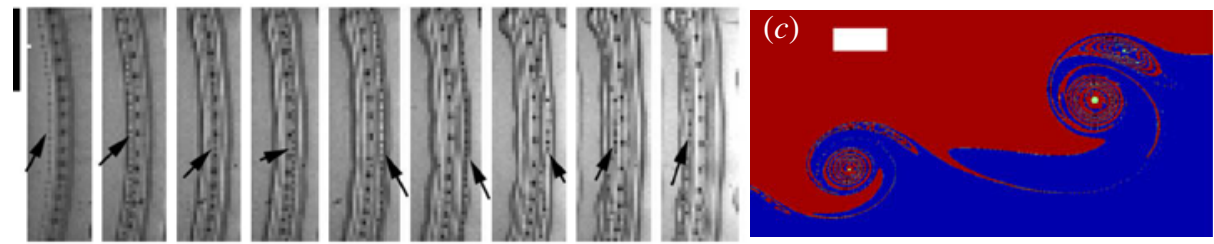

FIGURE 18. (a) Bubble rings formed during the impact of a water drop on a shallow pool of ethanol $(R e=13300, \alpha=0.98, \delta=250 \mu \mathrm{m})$. The frames are shown at $8,20,38,66$ and $112 \mu$ s after the first one, showing a total of seven bubble rings. (b) Close-up view of a line of fine bubbles which circulate around another line of slightly larger bubbles, from the movie in $(a)$. Total duration of these frames is $104 \mu \mathrm{s}$. See also supplementary movies. (c) Numerical simulation of a water drop impacting on a thin film of the same liquid $(\operatorname{Re}=13800, \alpha=1$, $\delta=800 \mu \mathrm{m})$. This focused view of the interface at $t^{*}=0.481$ shows the rotation of a pair of bubble tori at the core of adjacent vortex rings. The scale bars are all $100 \mu \mathrm{m}$ long.

\subsection{Three-dimensional instabilities}

The axisymmetry of the impact is rapidly broken by different instabilities (see figures 4 and 7). For a water pool, we have observed that undulations develop in the neck as soon as an ejecta forms (figure 4). This leads to entrapment of isolated bubbles (figures 4 and 5) and creates less regular bubble rings compared to ethanol or methanol pools. Even in those lower-surface-tension liquids, the most regular rings appear at smaller entrapment radii, where the ejecta sheet has not broken yet and remains axisymmetric. After the breakup of the ejecta sheet, the loss of axisymmetry is imprinted on the bubble entrapments, as shown by the bubble arcs observed in figure $7(f)$, with legs extending in the radial direction towards the neck.

We have already suggested in $\S 3.5$ that some of the azimuthal instabilities come from the breakup of the ejecta sheet, when it bends and touches the drop or pool surfaces. Overturning gravity waves also rebound and destabilize underlying vortices, but at a much larger scales than herein, see Watanabe, Saeki \& Hosking (2005). Figure 14 clearly demonstrates the effect of isolated neck disturbances on the bubble entrapment. A small perturbation is visible in the first frame and develops in time. The two first bubble rings which form in the following frames are broken at the same azimuthal location and the ejecta ruptures there first.

Three-dimensional instabilities also develop inside the liquid, and are made apparent by the difference in refractive index. Radial lines are visible in figures $7(e), 8,18(a)$, 19 and 20. They show the formation of streamwise vortices between the primary spanwise vortex rings, which often reach to the free surface in the neck. For the lower-Re cases (figure 19), isolated streamwise vortices are observed. In figure 19(a), they appear in pairs, at the same location as a front perturbation. The lower one in the 
(a)
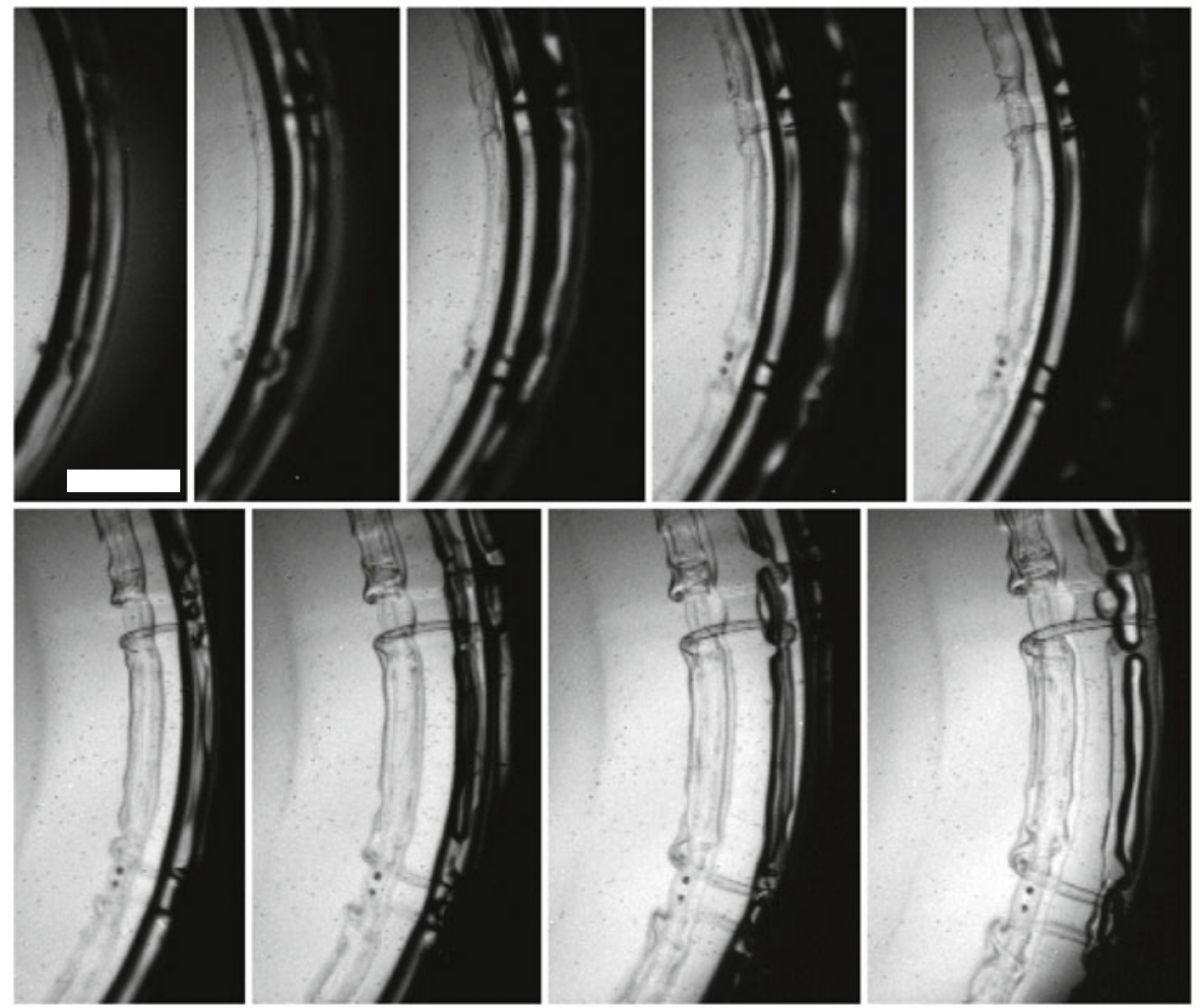

(b)

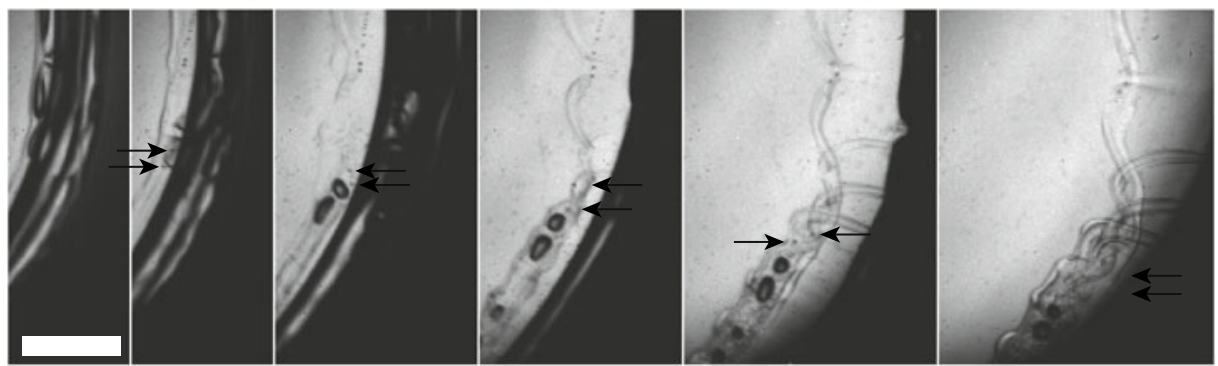

FIGURE 19. (a) Roll-up of isolated streamwise vortex pairs for a water drop impacting on a film of ethanol seeded with $2 \mu \mathrm{m}$ particles $(R e=11400, \alpha=1.05, \delta=125 \mu \mathrm{m})$. The first images show that the vortex pairs are starting at the same azimuthal location as local disturbances in the front. The bottom vortex pair first entraps a bubble, later splitting in two. Frames are shown 3, 6, 9, 12, 17, 23, 27 and $35 \mu$ s after the first one. (b) Roll-up and sideways motion of streamwise vortices for a water drop impacting on a film of ethanol seeded with $2 \mu \mathrm{m}$ particles $(R e=11400, \alpha=1.05, \delta=125 \mu \mathrm{m})$. Frames are shown 3, 10, 18, 32 and $47 \mu \mathrm{s}$ after the first one. The scale bars are $200 \mu \mathrm{m}$ long.

image starts at an isolated location on the side of a vortex ring, where it entraps one micro-bubble. Two lines are then visible on each side of this initial entrapment and extend up to the front. This suggests that the vortex pair arises from the same vortex loop, rolling-up around the vortex ring. The connection between the two streamwise vortex lines should then form a vortex loop with one section in the azimuthal direction, near its origin around the bubble. The presence of a strong vorticity around this 
(a)

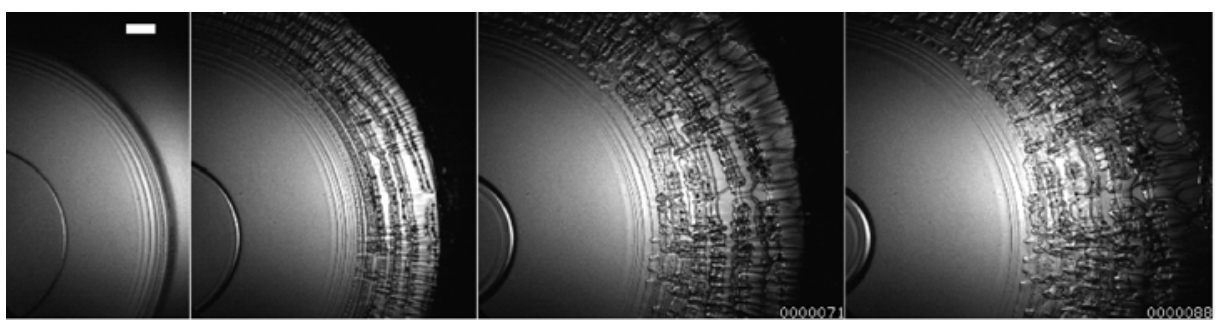

(b)

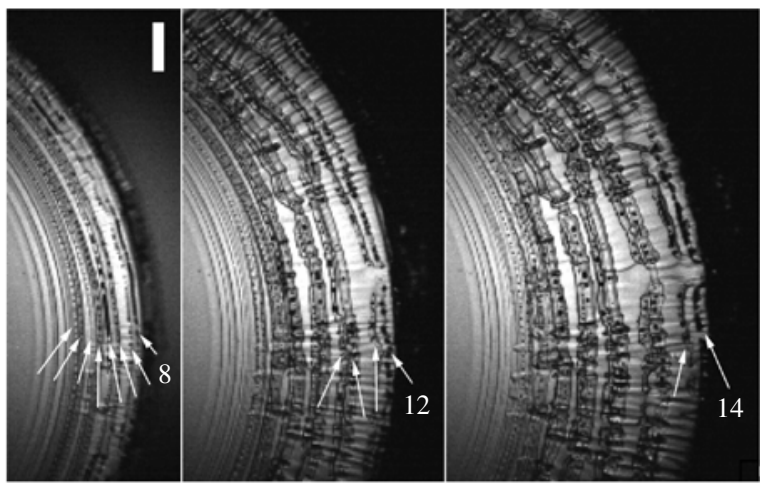

(c)

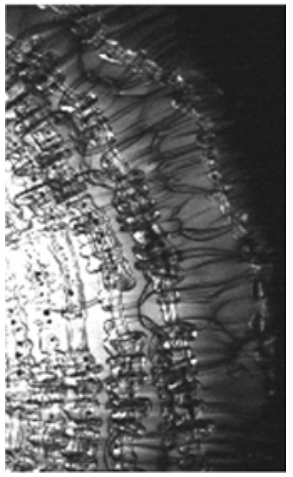

FIGURE 20. Bubble rings and vortex entanglement $(R e=12900, \alpha=0.80)$. (a) Frames shown are $8,22,41$ and $58 \mu$ s from first contact on ethanol $(\delta=250 \mu \mathrm{m})$. (b) Careful examination of the fine bubbles, shows 14 separate bubble rings, which are pointed out by the arrows. $(c)$ Close-up of the vortex tangles at $t=58 \mu \mathrm{s}$. The scale bars are $100 \mu \mathrm{m}$ long. See also supplementary movie.

bubble is demonstrated by its breakup into two smaller bubbles (in the 4th panel of figure 19a). Moreover, the later dynamics shows that the secondary streamwise vortex tubes roll up around the primary spanwise vortex ring. Figure 19(b) shows a similar case where streamwise vortices roll-up around a vortex ring. This roll-up can be identified by following two micro-bubbles at the core of the vortices (black arrows).

Interesting parallels can be made with similar three-dimensional instabilities occurring in the wake of a cylinder (Williamson 1996). The local Reynolds number at the base of the ejecta sheet $R e_{b}$ will be affected by both the radial velocity of the neck and the velocity within the liquid. $R e_{b}$ can be increased both by increasing the impact velocity or by using a more oblate drop. Indeed, as the impact $R e$ increases, the ejecta sheet emerges from a faster moving neck (Josserand \& Zaleski 2003; Thoraval et al. 2012), and at a higher velocity (Thoroddsen 2002; Josserand \& Zaleski 2003), thus leading to higher $R_{b}$. A flat-bottom drop also geometrically generates a faster moving neck, and produces larger velocities in the liquid, see figure 12(a). Both effects lead to a larger concentration of streamwise vortices (figures 7e, 8, 18a, 19 and 20). This is similar to the onset of three-dimensional instabilities of the vortex street behind a circular cylinder (Williamson 1988, 1996), where finer streamwise vortices and a smaller spanwise wavelength are observed at higher $R e$. Similar vortex loops are also observed in both cases, as described above. Moreover, rapid motion of the bubbles in the spanwise direction along the vortices is observed in our experiments, see figures $4(f)$ and $7(f)$. Similar lateral motion was also observed behind 'vortex dislocations' in the wake of a cylinder (Williamson 1992, 1996). 


\section{Conclusions}

Observations from below an impacting drop have herein demonstrated that the mechanism suggested in Thoraval et al. (2012) does indeed entrap micron-sized air tori. The oscillations of the ejecta sheet can thereby entrap a row of bubble rings. The vorticity entering the liquid during those oscillations and bubble entrapments is then destabilized into complex three-dimensional structures. The combination of azimuthal instabilities and vertical oscillations produces a large range of new bubble-entrapment scenarios.

Besides imaging the formation and breakup of partial bubble rings, of equal significance is our observation that for water-on-water impacts the outer neck is unstable to azimuthal undulations at even very moderate Reynolds numbers (figures $4 d$ and 5). This poses a challenge to theoretical and numerical studies, which invariably assume axisymmetry.

We note that the bubble rings observed herein differ in fundamental ways from the Oguz-Prosperetti rings (Oguz \& Prosperetti 1989), as the base of the ejecta is not driven by surface tension, but rather by the impact pressure. This high localized pressure is indeed the mechanism responsible for driving out the ejecta sheet.

However, the details of the air entrapment and its dependence on the impact conditions is still not clear. Large scope exists for further work, as the present study perhaps raises as many questions as it answers. What role do Marangoni or Rayleigh-Taylor instabilities play in the two-liquid dynamics? The large parameter space of other liquids needs to be studied. Even for the same liquids in the drop and pool, the interplay between $R e$, We and $\alpha$ which allows bubble-ring entrapment, or preserves an extended axisymmetric ejecta, remains to be determined. The threedimensional instabilities of the vortex street also need to be studied in more detail and compared to the well-known instabilities of the cylinder wake and the shear layer. Furthermore, in the vortex street observed here, the vortices are shed from a deformable free surface, adding to the complexity of the analysis. The influence of the shed vorticity on the dynamics of the neck, both vertically and in the azimuthal direction, should also be added to the factors influencing the stability analysis of splashing.

\section{Supplementary movies}

Supplementary movies are available at http://dx.doi.org/10.1017/jfm.2013.147.

\section{REFERENCES}

Agbaglah, G., Delaux, S., Fuster, D., Hoepffner, J., Josserand, C., Popinet, S., Ray, P., SCARdovelli, R. \& ZALESKI, S. 2011 Parallel simulation of multiphase flows using octree adaptivity and the volume-of-fluid method. C. R. Mec. 339 (2-3), 194-207.

Ashmore, J. \& Stone, H. A. 2004 Instability of a rotating thread in a second immiscible liquid. Phys. Fluids 16 (1), 29-38.

Castrejón-Pita, A. A., Castrejón-Pita, J. R. \& Hutchings, I. M. 2012 Experimental observation of von Kármán vortices during drop impact. Phys. Rev. E 86 (4), 045301(R).

Chandrasekhar, S. 1961 Hydrodynamic and Hydromagnetic Stability. Dover.

Czerski, H., Twardowski, M., Zhang, X. \& Vagle, S. 2011 Resolving size distributions of bubbles with radii less than $30 \mu \mathrm{m}$ with optical and acoustical methods. J. Geophys. Res. 116, C0OH11.

DaVidson, M. R. 2002 Spreading of an inviscid drop impacting on a liquid film. Chem. Engng Sci. 57 (17), 3639-3647. 
Driscoll, M. M. \& NAgel, S. R. 2011 Ultrafast interference imaging of air in splashing dynamics. Phys. Rev. Lett. 107 (15), 154502.

Eggers, J. \& Villermaux, E. 2008 Physics of liquid jets. Rep. Prog. Phys. 71 (3), 036601.

Etoh, T. G., Poggemann, D., Kreider, G., Mutoh, H., Theuwissen, A. J. P., Ruckelshausen, A., Kondo, Y., Maruno, H., Takubo, K., Soya, H., Takehara, K., OKINAKA, T. \& TAKANO, Y. 2003 An image sensor which captures 100 consecutive frames at 1000000 frames/s. IEEE Trans. Electron Devices 50 (1), 144-151.

GunN, R. \& KinZER, G. D. 1949 The terminal velocity of fall for water droplets in stagnant air. J. Meteorol. 6 (4), 243-248.

Hicks, P. D. \& PURVIS, R. 2010 Air cushioning and bubble entrapment in three-dimensional droplet impacts. J. Fluid Mech. 649, 135-163.

Howison, S. D., Ockendon, J. R., Oliver, J. M., Purvis, R. \& Smith, F. T. 2005 Droplet impact on a thin fluid layer. J. Fluid Mech. 542, 1-23.

Josserand, C. \& ZALeski, S. 2003 Droplet splashing on a thin liquid film. Phys. Fluids 15 (6), $1650-1657$.

Kiger, K. T. \& DunCAN, J. H. 2012 Air-entrainment mechanisms in plunging jets and breaking waves. Annu. Rev. Fluid Mech. 44, 563-596.

Kolinski, J. M., Rubinstein, S. M., Mandre, S., Brenner, M. P., Weitz, D. A. \& MAHADEVAn, L. 2012 Skating on a film of air: drops impacting on a surface. Phys. Rev. Lett. 108 (7), 074503.

Korobkin, A. A., Ellis, A. S. \& Smith, F. T. 2008 Trapping of air in impact between a body and shallow water. J. Fluid Mech. 611, 365-394.

LAMB, H. 1975 Hydrodynamics, 6th edn. Dover.

LAsheras, J. C. \& CHOI, H. 1988 Three-dimensional instability of a plane free shear layer: an experimental study of the formation and evolution of streamwise vortices. J. Fluid Mech. 189, 53-86.

Liow, J. L. \& COLE, D. E. 2007 Bubble entrapment mechanisms during the impact of a water drop. In 16th Australasian Fluid Mechanics Conf., pp. 866-869. School of Engineering, The University of Queensland.

Mani, M., Mandre, S. \& Brenner, M. P. 2010 Events before droplet splashing on a solid surface. J. Fluid Mech. 647, 163-185.

Oguz, H. N. \& Prosperetti, A. 1989 Surface-tension effects in the contact of liquid surfaces. J. Fluid Mech. 203, 149-171.

PeCK, B. \& Sigurdson, L. 1994 The three-dimensional vortex structure of an impacting water drop. Phys. Fluids 6 (2), 564-576.

PopINET, S. 2003 Gerris: a tree-based adaptive solver for the incompressible Euler equations in complex geometries. J. Comput. Phys. 190 (2), 572-600.

Popinet, S. 2009 An accurate adaptive solver for surface-tension-driven interfacial flows. J. Comput. Phys. 228 (16), 5838-5866.

Pumphrey, H. C., Crum, L. A. \& BJøRnØ, L. 1989 Underwater sound produced by individual drop impacts and rainfall. J. Acoust. Soc. Am. 85 (4), 1518-1526.

RaYleigh, Lord 1879 On the capillary phenomena of jets. Proc. R. Soc. Lond. 29 (196-199), 71-97.

Roisman, I., Gambaryan-Roisman, T., Kyriopoulos, O., Stephan, P. \& Tropea, C. 2007 Breakup and atomization of a stretching crown. Phys. Rev. E 76 (2), 026302.

Rosenthal, D. K. 1962 The shape and stability of a bubble at the axis of a rotating liquid. J. Fluid Mech. 12 (3), 358-366.

SAYLOR, J. R. \& GRIZZARD, N. K. 2004 The optimal drop shape for vortices generated by drop impacts: the effect of surfactants on the drop surface. Exp. Fluids 36 (5), 783-790.

Thoraval, M.-J., Takehara, K., Etoh, T. G., Popinet, S., Ray, P., Josserand, C., ZALESKI, S. \& THORODDSEN, S. T. 2012 von Kármán vortex street within an impacting drop. Phys. Rev. Lett. 108 (26), 264506.

Thoroddsen, S. T. 2002 The ejecta sheet generated by the impact of a drop. J. Fluid Mech. 451, 373-381.

Thoroddsen, S. T., Eтoh, T. G. \& TAKehara, K. 2003 Air entrapment under an impacting drop. J. Fluid Mech. 478, 125-134. 
Thoroddsen, S. T., Etoh, T. G. \& Takehara, K. 2008 High-speed imaging of drops and bubbles. Annu. Rev. Fluid Mech. 40, 257-285.

Thoroddsen, S. T., Takehara, K. \& Etoh, T. G. 2012 Micro-splashing by drop impacts. J. Fluid Mech. 706, 560-570.

Thoroddsen, S. T., Thoraval, M.-J., Takehara, K. \& Etoh, T. G. 2011 Droplet splashing by a slingshot mechanism. Phys. Rev. Lett. 106 (3), 034501.

van der Veen, R. C. A., Tran, T., Lohse, D. \& Sun, C. 2012 Direct measurements of air layer profiles under impacting droplets using high-speed color interferometry. Phys. Rev. E 85 (2), 026315.

Wanninkhof, R., Asher, W. E., Ho, D. T., Sweeney, C. \& McGillis, W. R. 2009 Advances in quantifying air-sea gas exchange and environmental forcing. Annu. Rev. Mar. Sci. 1, 213-244.

Watanabe, Y., Saeki, H. \& Hosking, R. J. 2005 Three-dimensional vortex structure under breaking waves. J. Fluid Mech. 545, 291-328.

WeIss, D. A. \& YARIN, A. L. 1999 Single drop impact onto liquid films: neck distortion, jetting, tiny bubble entrainment, and crown formation. J. Fluid Mech. 385, 229-254.

Williamson, C. H. K. 1988 The existence of two stages in the transition to three-dimensionality of a cylinder wake. Phys. Fluids 31 (11), 3165-3168.

Williamson, C. H. K. 1992 The natural and forced formation of spot-like 'vortex dislocations' in the transition of a wake. J. Fluid Mech. 243, 393-441.

Williamson, C. H. K. 1996 Vortex dynamics in the cylinder wake. Annu. Rev. Fluid Mech. 28, 477-539.

YARIN, A. L. 2006 Drop impact dynamics: splashing, spreading, receding, bouncing... Annu. Rev. Fluid Mech. 38, 159-192.

Zhang, L. V., Toole, J., FezzaA, K. \& Deegan, R. D. 2012 Evolution of the ejecta sheet from the impact of a drop with a deep pool. J. Fluid Mech. 690, 5-15. 